\title{
Review
}

\section{Abscisic acid perception and signaling: structural mechanisms and applications}

\author{
Ley Moy $\mathrm{NG}^{1, *}$, Karsten MELCHER ${ }^{2}$, Bin Tean $\mathrm{TEH}^{1,3,4}$, H Eric XU ${ }^{2,5}$ \\ ${ }^{1}$ Cancer Science Institute of Singapore, National University of Singapore, Singapore 117599, Singapore; ${ }^{2}$ Laboratory of Structural \\ Sciences, Center for Structural Biology and Drug Discovery, Van Andel Research Institute, Grand Rapids, MI 49503, USA; ${ }^{3}$ Laboratory \\ of Cancer Epigenome, Division of Medical Sciences, National Cancer Centre Singapore, Singapore; ${ }^{4}$ Division of Cancer and Stem Cell \\ Biology, Duke-National University of Singapore Graduate Medical School, Singapore; ${ }^{5}$ VARI-SIMM Center, Center for Structure and \\ Function of Drug Targets, Key Laboratory of Receptor Research, Shanghai Institute of Materia Medica, Chinese Academy of Sciences, \\ Shanghai 201203, China
}

\begin{abstract}
Adverse environmental conditions are a threat to agricultural yield and therefore exert a global effect on livelihood, health and the economy. Abscisic acid ( $A B A)$ is a vital plant hormone that regulates abiotic stress tolerance, thereby allowing plants to cope with environmental stresses. Previously, attempts to develop a complete understanding of the mechanisms underlying ABA signaling have been hindered by difficulties in the identification of bona fide ABA receptors. The discovery of the PYR/PYL/RCAR family of $A B A$ receptors therefore represented a major milestone in the effort to overcome these roadblocks; since then, many structural and functional studies have provided detailed insights into processes ranging from ABA perception to the activation of ABA-responsive gene transcription. This understanding of the mechanisms of ABA perception and signaling has served as the basis for recent, preliminary developments in the genetic engineering of stress-resistant crops as well as in the design of new synthetic ABA agonists, which hold great promise for the agricultural enhancement of stress tolerance.
\end{abstract}

Keywords: abscisic acid; abiotic stress; ABA receptor; plant hormone

Acta Pharmacologica Sinica (2014) 35: 567-584; doi: 10.1038/aps.2014.5

\section{Introduction-Understanding ABA perception}

"There are things known and there are things unknown, and in between are the doors of perception."

- Aldous Huxley

We are constantly surrounded by signals, such as traffic signals, notifications, advertisements, feelings of pain or hunger. However, we are only aware of these signals if we perceive them; we would not know of or respond to these signals if we could not perceive them, even if the signals are always there. Thus, in the process of conveying a signal, the perception of the signal is just as important as the existence of the signal itself.

At the cellular level, extracellular signals are often transmitted to cells via molecules such as hormones. In plants, the levels of the hormone abscisic acid (ABA) increase when environmental conditions are harsh and serve as a signal for

\footnotetext{
* To whom correspondence should be addressed.

E-mail csinlml@nus.edu.sg

Received 2013-12-23 Accepted 2014-01-16
}

the plant cells to adapt as necessary. ABA was discovered in the 1960s, and many details were established shortly thereafter regarding the chemistry and physiological importance of $\mathrm{ABA}^{[1]}$. Over the ensuing decades, more than 100 mediators involved in ABA signaling have been identified using molecular genetic, biochemical and pharmacological studies ${ }^{[2]}$. However, for almost half a century, a critical piece of the puzzle remained missing in our knowledge of ABA signal transduction, namely, how it is that ABA signals are perceived.

The identification of receptors capable of sensing ABA and relaying this signal to other mediators in the pathway has proven to be a daunting task rife with controversy and frustration. Since 2006, several reports have claimed to identify the $\mathrm{ABA}$ receptors ${ }^{[3]}$, but none of these reports could be substantiated after further investigation. The turnaround came in 2009, when at least two separate findings convincingly implicated the same members of the steroidogenic acute regulatory protein (StAR)-related lipid-transfer (START) domain/ major birch pollen allergen (Bet $\mathrm{v} 1$ ) superfamily of proteins as candidate ABA receptors ${ }^{[4-7]}$. The 14 members of this group of proteins are named Pyrabactin Resistance 1 (PYR1) and 
PYR1-like 1-13 (PYL1-PYL13) ${ }^{[6]}$ or Regulatory Component of ABA Receptor (RCAR1-RCAR14) ${ }^{[4]}$ and are referred to in this article as PYL(s) for simplicity. The initial discovery of PYLs soon led to a series of crystallographic studies that collectively unraveled the mechanistic details underlying ABA recognition and signal transduction by the members of this family of ABA receptors. These findings have fuelled remarkable advancements in the field of ABA signaling and hence were recognized as both one of the scientific breakthroughs ${ }^{[8]}$ and one of the signaling breakthroughs ${ }^{[9]}$ of the year for 2009.

Since then, the molecular interactions comprising the core ABA signaling pathway, a signaling cascade consisting of the PYL ABA receptors, type $2 \mathrm{C}$ protein phosphatases (PP2Cs) and Snf1-related protein kinases 2 (SnRK2s), have been further clarified by multiple structural studies. Studies such as these shed light onto the exquisite controls regulating each level of the ABA signal transduction pathway and open up new technological opportunities for the enhancement of abiotic stress tolerance in agriculture. Here, we present a comprehensive review of the current understanding of ABA, its early signaling mechanisms and recent developments in agricultural applications based on the emerging structural findings.

\section{ABA in plants}

\section{Physiological role in abiotic stress tolerance}

ABA is a vital phytohormone that confers abiotic stress tolerance in plants ${ }^{[10]}$. Under stressful environmental conditions such as water shortage, high salinity and temperature extremes, the ABA content in plants rises significantly, stimulating stress-tolerance effects that help plants adapt and survive under these adverse conditions (Figure 1). It has been demonstrated that mutant plants engineered with enhanced ABA sensitivity adapt to drought conditions better than their wild-type counterparts ${ }^{[11]}$. Under drought or osmotic stress conditions, ABA promotes stomatal closure, which prevents water loss through transpiration, and the accumulation of osmocompatible solutes to retain water ${ }^{[2,12]}$. It has also been shown that $\mathrm{ABA}$ is required for tolerance to freezing which occurs through the induction of dehydration-tolerance genes ${ }^{[13]}$.

The role of $\mathrm{ABA}$ as a negative regulator of plant growth has also been long established ${ }^{[14]}$. The activity of $\mathrm{ABA}$ in the induction and maintenance of seed dormancy is attributed to its potent effects on the inhibition of seed germination ${ }^{[15]}$. ABA also inhibits the growth and development of whole plants or plant parts and counteracts the effects of growth-stimulating hormones such as gibberellins ${ }^{[2]}$. The inhibitory effects of ABA on germination and growth help plants withstand these stressful conditions and germinate only when the conditions are favorable for growth.

\section{Biosynthesis and transport}

Stress signals induce the expression of enzymes responsible for ABA biosynthesis. Most of the ABA biosynthetic genes have been identified and cloned, including zeaxanthin epoxidase (ZEP), 9-cis-epoxycarotenoid dioxygenase (NCED) and

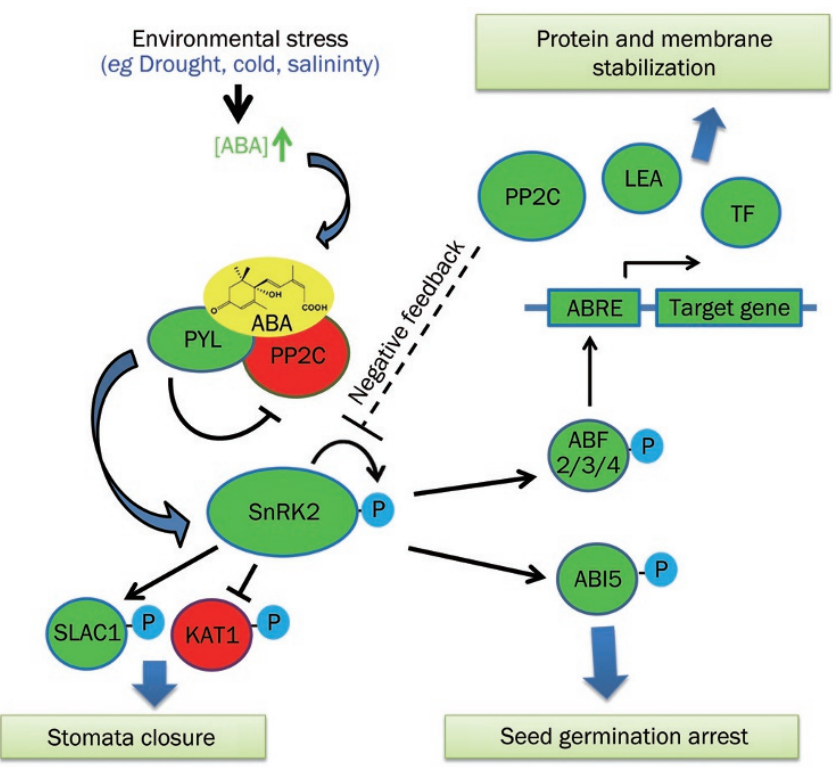

Figure 1. ABA-mediated abiotic stress response. Overview of the molecular events in ABA-mediated stress tolerance. ABA accumulation, induced by stress signals, activates PYL ABA receptors to inhibit group A PP2Cs. PP2C inhibition in turn allows SnRK2 activation through autophosphorylation. Active $S n R K 2 s$ mediate the ABA response through the phosphorylation of downstream targets. In guard cells, SnRK2s phosphorylate the SLAC1 and KAT1 ion channels, resulting in stomatal closure to prevent transpirational water loss. In seeds, ABI5 phosphorylation by SnRK2s leads to the inhibition of seedling growth. The phosphorylation of the AREB1 (ABF2), AREB2 (ABF4), and ABF3 transcription factors activates the transcription of target genes such as Late Embryogenesis Abundant (LEA)-class genes as well as transcription factors (TF) involved in stress tolerance. Transcriptional increases in the expression of group A PP2C genes may function as a negative feedback loop in the ABA response pathway by inhibiting SnRK2 activity. Positive regulators of the $A B A$ signaling pathway are shown in green, whereas negative regulators are shown in red.

abscisic aldehyde oxidase $(A A O 3)^{[13,16]}$. The synthesis of ABA from $\mathrm{C}_{40}$ carotenoids occurs via several enzymatic steps (Figure 2$)^{[17]}$. First, zeaxanthin, which is formed by the hydroxylation of $\beta$-carotene, is converted into violaxanthin by ZEP. This is followed by the synthesis and oxidative cleavage of neoxanthin into xanthoxin, the $C_{15}$ precursor of $A B A$. The NCED-catalyzed production of xanthoxin is thought to be the key regulatory step of ABA biosynthesis. Xanthoxin is then converted into abscisic aldehyde, which is oxidized into ABA.

The endogenous concentration of $\mathrm{ABA}$ is determined by the balance between ABA biosynthesis and catabolism, as well as by the rate of ABA transport to its sites of action. Although ABA can diffuse passively across biological membranes, recent evidence suggests that transport proteins are involved in actively shuttling ABA in and out of cells. In 2010, two ATP-binding cassette $(A B C)$ transporters were found to be capable of shuttling ABA: AtABCG25 is mainly expressed in vascular tissues, where ABA is predominantly synthesized, and can function as an ABA exporter ${ }^{[18]}$, whereas AtABCG40 is 


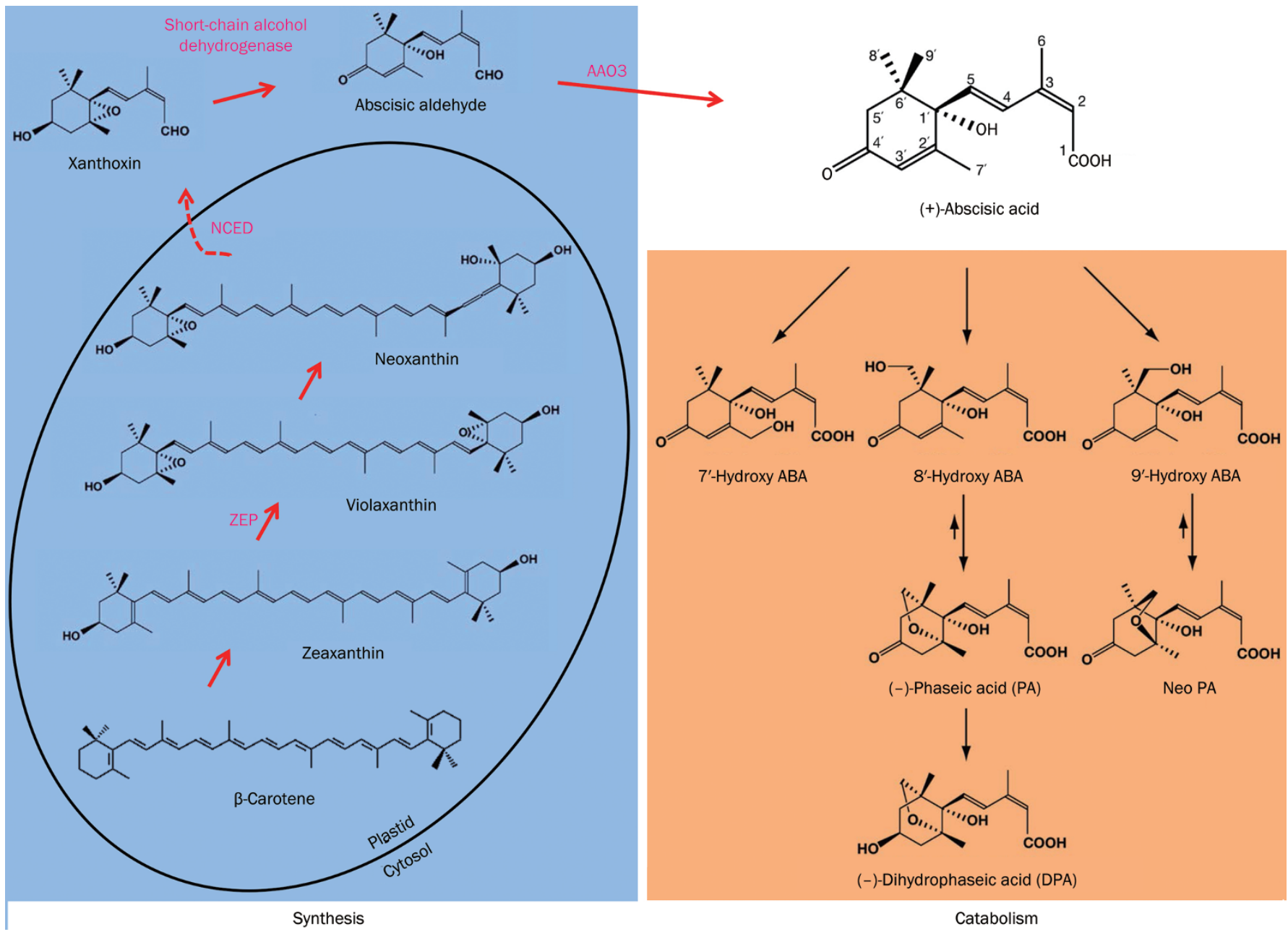

Figure 2. ABA biosynthesis and catabolism. The ABA biosynthetic pathway is shown in the left panel. ABA is derived from $C_{40}$ epoxycarotenoid precursors through oxidative cleavage reactions that occur in the plastid. The $C_{15}$ intermediate xanthoxin is exported into the cytosol, where it is converted to ABA through a two-step reaction involving an abscisic aldehyde intermediate. The ABA biosynthetic enzymes zeaxanthin epoxidase (ZEP), 9-cis-epoxycarotenoid dioxygenase (NCED), short-chain alcohol dehydrogenase and abscisic aldehyde oxidase (AAO3) are shown in pink. In the right panel, three catabolic pathways involving $C-7^{\prime}, C-8^{\prime}$, and $C-9^{\prime}$ hydroxylation are shown. ABA is fully inactivated after the formation of the biologically inactive compound DPA.

expressed at the sites of ABA action, especially in guard cells, and can function as an ABA importer ${ }^{[19]}$. Thus, a simple model of ABA translocation was proposed ${ }^{[20]}$ in which ABA is synthesized in vascular cells and actively transported into the extracellular apoplastic space by AtABCG25, after which it is taken up by roots and leaves via AtABCG40. However, whereas AtABCG40 is required for efficient ABA signaling as expected, ABA responses are increased and decreased upon AtABCG25 loss-of-function and overexpression, respectively, which cannot be easily explained by this model. Moreover, a second, unrelated ABA importer, ABA-importing transporter 1 (AIT1), has recently been identified ${ }^{[21]}$ and unexpectedly appears to be expressed predominantly at the sites of ABA synthesis, indicating that $\mathrm{ABA}$ transport may be more complex than initially envisioned.

\section{Catabolism}

When stress signals are attenuated, ABA is metabolized into inactive products. ABA catabolism occurs largely through the hydroxylation of one of the three methyl groups (C-7', C-8',

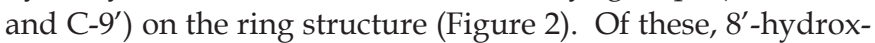
ylation is thought to serve as the dominant ABA catabolic pathway ${ }^{[22,23]}$. Accordingly, the products of the $8^{\prime}$-hydroxylation pathway, phaseic acid (PA) and dihydrophaseic acid (DPA), are the most abundant ABA catabolites. Furthermore, while 8'-hydroxy ABA exhibits substantial biological activity, the spontaneous cyclization of 8 -hydroxy ABA into PA causes a significant reduction in activity ${ }^{[24]}$. PA is further catabolized into the biologically inactive compound DPA.

ABA 8'-hydroxylation is catalyzed by ABA 8'-hydroxylase, a cytochrome P450 encoded by the CYP707A gene family ${ }^{[25,26]}$. 
Transcript levels for the four CYP707A family members, CYP707A1-CYP707A4, increase during drought stress and are strongly induced upon rehydration ${ }^{[25]}$, with CYP707A3 showing the highest induction ${ }^{[27]}$. Recent findings indicated that CYP707A3 functions in vascular tissues to reduce systemic ABA levels, whereas CYP707A1 catabolizes local ABA pools inside guard cells in response to high humidity ${ }^{[23]}$.

In addition to hydroxylation, $\mathrm{ABA}$ and its hydroxylated catabolites can be conjugated to glucose. The major glucose conjugate of ABA, ABA glucosyl ester (ABA-GE), is biologically inactive and may function as a storage molecule to form a releasable pool of $\mathrm{ABA}^{[28]}$. ABA-GE is presumably synthesized in the cytosol and stored in the vacuoles ${ }^{[29]}$. The import of ABA-GE into vacuoles has been reported to be mediated by proton gradient-driven and ABC-type transporters ${ }^{[30]}$. Under abiotic stress conditions, ABA-GE is hydrolyzed by $\beta$-glucosidases to release free $\mathrm{ABA}^{[28,31,32]}$.

\section{Chemical features}

The natural and biologically active isomer of ABA is (+)-2-cis4-trans-ABA, also commonly known as S-ABA, $(+)-\mathrm{ABA}$ or $S-(+)-A B A$. The molecular structure consists of a cyclohexene ring with a monomethyl group, a dimethyl group, a ketone group, a hydroxyl group and a hydrocarbon side chain conjugated to the carboxylic acid group (Figure 3A). The 2-cis4-trans side chain geometry can be reversibly isomerized by light to form a mixture containing the inactive 2-trans-4-trans isomer ${ }^{[2]}$. Studies using ABA analogs lacking the $7^{\prime}-, 8^{\prime}-$, or $9^{\prime}$ methyl groups showed that the $7^{\prime}$-methyl group is critical for bioactivity ${ }^{[33]}$. Flipping the cyclohexene ring around the chiral carbon produces the unnatural (-)-enantiomer, which has weak biological activity (Figure 3B) ${ }^{[34]}$.

Structural and functional analogs of ABA have been used to identify ABA-responsive genes, and some of these analogs are potential plant growth regulators ${ }^{[35,36]}$. For instance, PBI51 (Figure 3C), which is structurally similar to ABA, exhibits

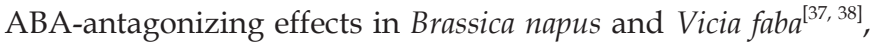

whereas it is a weak ABA agonist in Arabidopsis ${ }^{[39]}$. The use of PBI-51 to screen for ABA-related mutants has led to the isolation of novel ABA hypersensitive mutants ${ }^{[39]}$. In addition, pyrabactin (Figure 3D), a selective ABA agonist that does not structurally resemble $\mathrm{ABA}$, was employed in the isolation of PYR1, which led to the identification of the PYL family of proteins as ABA receptors ${ }^{[6]}$. Recent efforts to identify new ABA agonists based on structural studies of the activation of PYL by ABA and pyrabactin have led to the isolation of AM1/ quinabactin as a promising candidate (Figure $3 \mathrm{E})^{[40,41]}$. These studies will be reviewed in further detail in the later sections.

\section{Components of the ABA signaling pathway PYLs-ABA receptors}

Forward genetics approaches are most commonly used in the identification of hormone receptors, whereby mutants are identified based on their hormone-insensitive phenotypes. In the case of $\mathrm{ABA}$, the inhibition of seed germination by the application of exogenous $\mathrm{ABA}$ is the most widely used assay for mutational screening. These screens have identified several ABA-insensitive mutants ${ }^{[22,43]}$, five of which have been extensively characterized. These five mutants are designated abi1-abi5 (for ABA insensitive). The corresponding ABI1 and $A B I 2$ genes encode PP2Cs ${ }^{[44-46]}$ (see Section PP2Cs-negative regulators below), while $A B I 3, A B I 4$ and $A B I 5$ encode transcription factors involved in the seed-specific ABA signaling pathway ${ }^{[47-49]}$. None of the genes identified through classical forward genetics approaches had ABA-binding or receptorlike properties. Conversely, the use of alternative approaches has identified several putative ABA receptors, including $\mathrm{FCA}^{[50]}, \mathrm{CHLH}^{[51]}, \mathrm{GCR} 2^{[52]}$, GTG1 and GTG2 ${ }^{[53]}$, which all reportedly bind $\mathrm{ABA}$ with affinities in the nanomolar range $\left(K_{d}\right.$ of $19,32,21,35.8$, and $41.2 \mathrm{nmol} / \mathrm{L}$ for FCA, CHLH, GCR2, GTG1, and GTG2, respectively). These attempts have not, however, led to the unambiguous identification of convincing $\mathrm{ABA}$ receptor candidates that fit with the physiological, genetic and biochemical knowledge that has accumulated over
A

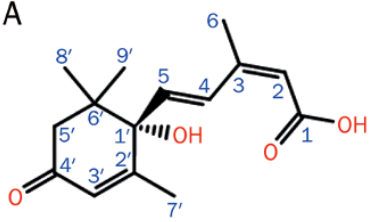

S-(+)-ABA

D

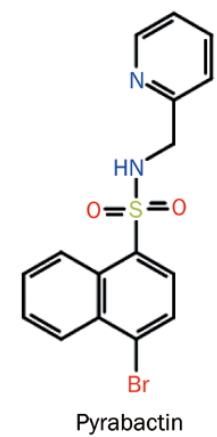

B

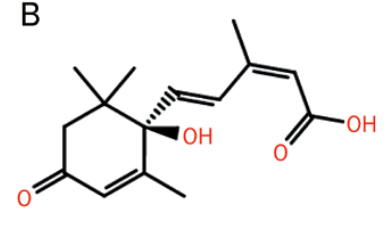

$R-(-)-\mathrm{ABA}$

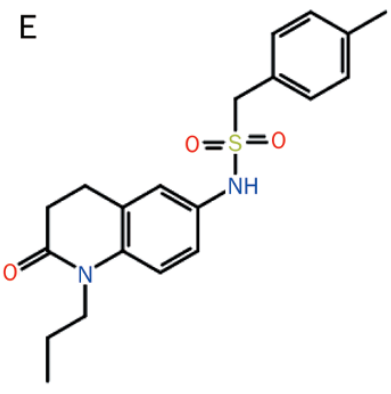

AM1/quinabactin

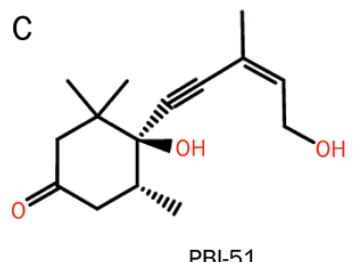

Figure 3. Chemical structures of ABA stereoisomers, structural analogs and pyrabactin. Chemical structures of $(A)$ the naturally occurring $S-(+)-$ ABA; $(B)$ the unnatural $R$-stereoisomer, which differs from the $S$-isomer by a flip around the chiral carbon (C-1'); (C) PBI-51, a structural analog of ABA; (D) Pyrabactin, a seed-specific selective ABA agonist that does not structurally resemble $A B A$; and $(E) A M 1$ /quinabactin, a recently identified $A B A$ agonist that has been shown to exhibit ABA-like effects in vegetative tissues. Functional groups for all molecules are shown in colors, and the nomenclature for the naming of carbon atom positions is shown for the S-(+)-ABA structure. 
the years ${ }^{[3]}$.

The discovery of PYLs as candidate ABA receptors was different from that of the earlier putative ABA receptors. Independent findings from several groups converged upon this novel class of ABA binding proteins, which fit elegantly into a model that connected the core components of the ABA signal transduction pathway.

Genetic redundancy has been one of the major issues hindering the identification of ABA receptors using classical forward genetics. To overcome this problem, Park et al used a synthetic seed germination inhibitor named pyrabactin as a selective ABA agonist ${ }^{[6]}$. Pyrabactin's effects on global gene transcription were highly correlated with those of ABA in seeds; however, the correlation was weaker in seedlings, indicating that pyrabactin is a highly selective ABA agonist that affects only a subset of ABA's activities. Forward genetic screens for pyrabactin-resistant mutants identified the Pyrabactin Resistance 1 (PYR1) locus, and 13 additional PYR1-like (PYL) genes were identified by sequence analysis and designated PYL1 through PYL13 (Table 1). Using yeast two-hybrid assays, the authors detected interactions between multiple PP2Cs and several PYL members in the presence of ABA. They also demonstrated that PYR1 inhibits the phosphatase activity of the PP2C HAB1 in the presence of $\mathrm{ABA}$, with an $\mathrm{IC}_{50}$ of $125 \mathrm{nmol} / \mathrm{L} \mathrm{ABA}$. The binding of ABA to PYR1 has also been detected in heteronuclear single quantum coherence (HSQC) nuclear magnetic resonance (NMR) experiments, which probe the chemical shifts of protein amide-NH bonds in response to ligands. Collectively, these data suggest that PYLs are ABA receptors that directly interact with and inhibit PP2Cs upon ABA binding.

Meanwhile, in a study to identify the link between ABA perception and PP2Cs, Ma et al performed a yeast two-hybrid screen for Arabidopsis proteins that interact with the PP2C $\mathrm{ABI} 2^{[4]}$. Using this approach, this group discovered an ABI2- interacting protein and named it Regulatory Component of ABA Response 1 (RCAR1). Subsequently, 13 homologous proteins were identified and named RCAR2 through RCAR14 (Table 1). The 14 RCAR members discovered in this study turned out to be identical to the 14 PYL members identified by Park et al. Using isothermal titration calorimetry (ITC), the interaction between RCAR1 and (+)-ABA was shown to possess an apparent $K_{d}$ of $\sim 0.66 \mu \mathrm{mol} / \mathrm{L}$. The binding affinity was approximately 10-fold higher in the presence of ABI2, suggesting the formation of a stable receptor-ABA-PP2C complex. RCAR1 was also shown to interact with and inhibit the phosphatase activities of the PP2Cs ABI1 and ABI2 in the presence of ABA, which is consistent with the ABA-binding and PP2Cinhibitory activities demonstrated by Park et al.

Using a similar approach, Santiago et al identified PYL5, PYL6, and PYL8 in a yeast two-hybrid screen for HAB1interacting proteins ${ }^{[7]}$. Consistent with the findings of Park et al and Ma et al, members of the PYL family inhibited the phosphatase activities of ABI1, ABI2 and HAB1 in an ABAdependent manner. Using ITC, the apparent $K_{d}$ of $(+)$-ABA binding to PYL5 was determined to be $1.1 \mu \mathrm{mol} / \mathrm{L}$ and 38 $\mathrm{nmol} / \mathrm{L}$ in the absence and presence of HAB1, respectively, supporting the formation of a stable ternary complex as suggested by Ma et al. In addition, Santiago et al observed that, whereas certain PYL members such as PYR1 and PYL4 only interacted with PP2Cs in the presence of ABA, PYL5, PYL6, and PYL8 did not require ABA to interact with HAB1 in a yeast two-hybrid assay, although ABA did enhance the affinity of PYL5 for HAB1. This finding suggests the existence of different roles for the PYL members such that a subset of PYL proteins may constitutively inhibit PP2Cs.

In yet another independent study, Nishimura et al screened for ABI1-interacting proteins in planta and identified 9 of the 14 PYL members using co-immunoprecipitation assays fol-

Table 1. List of 14 Arabidopsis PYL members, their oligomeric states and ABA-binding affinities.

\begin{tabular}{|c|c|c|c|c|c|c|c|}
\hline \multirow{2}{*}{\multicolumn{2}{|c|}{ PYL/RCAR }} & \multicolumn{2}{|c|}{ Oligomeric state } & \multirow[b]{2}{*}{ Without PP2C } & \multicolumn{3}{|c|}{ Apparent ABA binding affinities $\left(K_{d}, \mu \mathrm{mol} / \mathrm{L}\right)$} \\
\hline & & & Reference(s) & & Reference(s) & With PP2C & Reference(s) \\
\hline PYR1 & RCAR11 & Dimer & Hao et al, Dupeux et al & $97( \pm 36)$ & Dupeux et al & & \\
\hline PYL1 & RCAR12 & Dimer & Hao et al, Dupeux et al & 52 & Dupeux et al, Miyazono et al & & \\
\hline PYL2 & RCAR14 & Dimer & Hao et al & $59.1( \pm 2.5)$ & Yin et al & & \\
\hline PYL3 & RCAR13 & $\begin{array}{l}\text { Dimer } \\
\text { Monomer-dimer }\end{array}$ & $\begin{array}{l}\text { Zhang et al } \\
\text { Hao et al }\end{array}$ & & & & \\
\hline PYL4 & RCAR10 & Monomer & Hao et al & & & & \\
\hline PYL5 & RCAR8 & Monomer & Hao et al, Dupeux et al & $1.0( \pm 0.06)$ & Santiago et al & $0.038( \pm 0.006)$ & Santiago et a \\
\hline PYL6 & RCAR9 & Monomer & Hao et al, Dupeux et al & $1.1( \pm 0.01)$ & Dupeux et al & & \\
\hline PYL7 & RCAR2 & & & & & & \\
\hline PYL8 & RCAR3 & Monomer & Hao et al, Dupeux et al & & & & \\
\hline PYL9 & RCAR1 & Monomer & Hao et al & $\begin{array}{l}0.9( \pm 0.15) \\
0.66( \pm 0.08)\end{array}$ & $\begin{array}{l}\text { Dupeux et al, Szostkiewicz et al } \\
\text { Ma et al }\end{array}$ & $0.064( \pm 0.008)$ & Ma et al \\
\hline PYL10 & RCAR4 & Monomer & Hao et al & & & & \\
\hline PYL11 & RCAR5 & Monomer & Hao et al & & & & \\
\hline PYL12 & RCAR6 & Monomer & Hao et al & & & & \\
\hline PYL13 & RCAR7 & Monomer & Hao et al & & & & \\
\hline
\end{tabular}


lowed by mass spectrometry ${ }^{[5]}$. Co-immunoprecipitation experiments showed that $\mathrm{ABA}$ induces an interaction between PYR1 and ABI1 in vivo within 5 min of ABA treatment.

\section{PP2Cs-negative regulators}

Reversible protein phosphorylation mediated by protein kinases and protein phosphatases is a major and universal component of cellular signal transduction. PP2Cs are a group of single subunit $\mathrm{Mg}^{2+} / \mathrm{Mn}^{2+}$-dependent Ser/Thr phosphatases, and PP2Cs represent a major phosphatase family in plants. Of the 112 phosphatases encoded in the Arabidopsis genome, 76 are PP2Cs, which genetically cluster into 10 groups (A-J), with the exception of 6 genes that could not be clustered $^{[54]}$. At least 6 of the 9 members of the group A PP2Cs have been shown to be involved in ABA signaling, and of these, ABI1, ABI2, and HAB1 are the most well characterized.

The isolation and characterization of ABA-insensitive Arabidopsis mutants have led to the identification of group A PP2Cs as negative regulators of ABA signaling. The dominant mutants abi1-1 (ABI1 G180D) and abi2-1 (ABI2 G168D), which result in reduced $\mathrm{ABA}$ responsiveness, were isolated from genetic screens of mutagenized Arabidopsis seeds ${ }^{[44-46,55]}$. Both of these mutants display reduced seed dormancy, seedling growth, drought tolerance and stomatal regulation. The ABI1 and $A B I 2$ genes encode homologous PP2C proteins and are transcriptionally upregulated by $\mathrm{ABA}$ treatment. HAB1 was subsequently identified based on its sequence homology to $A B I 1$ and $A B I 2^{[56]}$.

Consistent with the phenotypic effects observed in the abi1-1 and abi2-1 mutants, introducing the corresponding mutation (G246D) into HAB1 resulted in strong ABA insensitivity ${ }^{[57]}$. The abi1-1, abi2-1, and hab1 G246D mutations correspond to the substitution of a conserved glycine residue with an aspartate in the phosphatase catalytic center. These mutants exhibited dramatically reduced phosphatase activity toward phospho-casein, a heterologous substrate, which was difficult to reconcile with the apparent dominant nature of the mutation. Recessive loss-of-function mutations in the catalytic regions of ABI1, ABI2, and HAB1 resulted in ABA-hypersensitive phenotypes (partially constitutive seed dormancy and drought tolerance), indicating that the ABA insensitivity of the abi1-1 and abi2-1 mutations is due not to the observed reduction in catalytic activity but to the gain of a new function, and provided critical evidence that these PP2Cs negatively regulate ABA signaling ${ }^{[58-60]}$. This notion was further confirmed using double- or triple-PP2C-knockout mutants, which display constitutively enhanced stress responses/ABA hypersensitivity ${ }^{[11,61]}$. Consistent with this, the overexpression of HAB1 (35S:HAB1) in Arabidopsis led to reduced ABA sensitivity, supporting the role of $\mathrm{HAB}$ as an inhibitor of ABA signaling ${ }^{[58]}$.

Given the negative regulatory roles of the group A PP2Cs, the mechanism by which the abi1-1 and abi2-1 mutations, as well as the corresponding G246D mutation in HAB1, induce dominant ABA-insensitive phenotypes remains an enigma. Interestingly, the mutant abi1-1 protein was as effective at inhibiting ABA signal transduction as the wild-type protein, as shown with an ABA-inducible transcription assay in plant protoplasts ${ }^{[62]}$. Although earlier studies indicated the hypermorphic nature of this type of mutation ${ }^{[57,63]}$, they were unable to fully explain how such a mutation interferes with ABA signal transduction. Instead, the answer lies in the loss of the ABA receptor-mediated regulation of PP2Cs, as explained by more recent structural findings.

\section{SnRK2s-positive mediators}

The identification of PP2Cs indicated that protein phosphorylation events are important in ABA signaling. In line with this concept, the SnRK2 family was identified as group of ABAactivated protein kinases ${ }^{[64,65]}$. SnRK2s belong to the SnRK group of protein kinases that are closely related to yeast Snf1 and to the catalytic subunits of mammalian AMPK. The Arabidopsis genome contains 38 SnRKs, which are classified into three groups, namely, SnRK1 (1.1-1.3), SnRK2 (2.1-2.10) and SnRK3 (3.1-3.25) ${ }^{[66]}$ (Figure 4A). The SnRK1 group shares the highest degree of homology with Snf1 and AMPK. Similar to its yeast and mammalian counterparts, SnRK1 is best known for its role as a key metabolic regulator ${ }^{[67]}$. In contrast, SnRK2 and SnRK3 are unique to plants and are thought to be involved in abiotic stress signaling ${ }^{[68]}$.

There are 10 SnRK2 members in Arabidopsis, which are designated as SRK2I-SRK2 ${ }^{[65]}$ or SnRK2.1-SnRK2.10 ${ }^{[66]}$ and divided into three subclasses: I, II and III (Figure 4A). All SnRK2 members, except for SnRK2.9, can be activated by osmotic stress, as shown in an Arabidopsis protoplast system ${ }^{[69]}$. Consistent with this observation, an Arabidopsis decuple mutant lacking all $10 \mathrm{SnRK2s}$ grew poorly under osmotic stress conditions ${ }^{[70]}$, revealing the importance of SnRK2s in osmotic stress signaling. However, not all SnRK2 members can be activated by $A B A$, suggesting that osmotic stress signaling consists of ABA-dependent and ABA-independent pathways. Whereas SnRK2 subclass I members are not activated by ABA, subclass II members, represented by SnRK2.7 and SnRK2.8, are weakly activated by ABA. In contrast, the members of subclass III are strongly activated by $\mathrm{ABA}^{[69]}$.

Subclass III of the Arabidopsis SnRK2 family contains three kinases, namely, SnRK2.2/SRK2D, SnRK2.3/SRK2I, and SnRK2.6/SRK2E/OST1. The members of this subclass of ABA-responsive kinases have been identified as the main positive regulators of ABA signaling. The physiological role of SnRK2.6 has been initially determined in guard cells. Lossof-function mutations in SnRK2.6 disrupted ABA-induced stomatal closure in Arabidopsis ${ }^{[64,65]}$. On the other hand, a snrk2.2 snrk2.3 double mutant showed strong ABA insensitivity with respect to seed germination and root growth inhibition ${ }^{[71]}$. Consequently, triple mutants lacking SnRK2.2, SnRK2.3 and SnRK2.6 were impaired in almost all ABA responses, indicating the centrality of these kinases to ABA signaling ${ }^{[72-74]}$.

SnRK2s contain a well-conserved kinase catalytic domain and a C-terminal regulatory region that encompasses two domains. Domain I, which is also known as the SnRK2 box, is conserved in all SnRK2s and is required for kinase activity. Domain II is a highly acidic region that is important for PP2C 
A

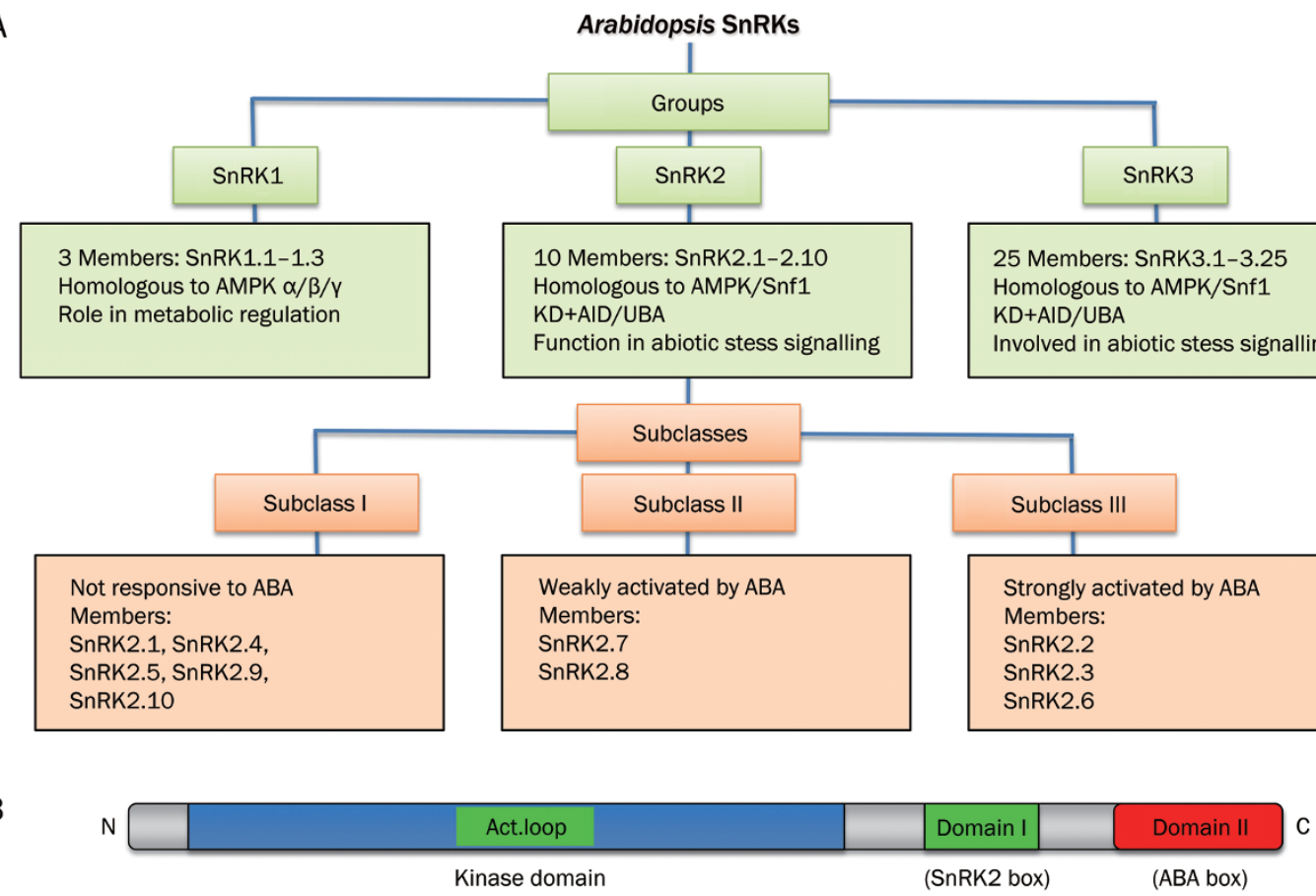

Figure 4. Classification and domain structure of Arabidopsis SnRK2. (A) Diagram showing the classification of Arabidopsis SnRK groups and the SnRK2 subclasses. (B) Domain structure of Arabidopsis SnRK2s.

interaction and is only conserved among the ABA-responsive members; it is thus also known as the ABA box ${ }^{[75,76]}$ (Figure 4B).

Many reports have demonstrated that phosphorylation is important for the activation of SnRK2s. Active recombinant SnRK2.6 is autophosphorylated, and several phosphorylation sites have been identified, with the phosphorylation of S175 within the activation loop shown to be critical for kinase activity $^{[75]}$. Active SnRK2s are able to directly phosphorylate target proteins such as ion channels and transcription factors to elicit the $\mathrm{ABA}$ response.

ABA-induced stomatal closure appears to be mediated by ion channels on guard cell membranes. One of these is the slow-type anion channel SLAC1, which has been shown to be essential for stomatal closure in response to various factors such as ABA and $\mathrm{CO}_{2}{ }^{[77,78]}$. SLAC1 is phosphorylated and activated by SnRK2.6, a process that can be inhibited by $\mathrm{PP} 2 \mathrm{C}^{[79,80]}$. In addition, an inward-rectifying potassium channel, KAT1, is also a target of SnRK2.6. ABA-activated SnRK2.6 can phosphorylate T306 in KAT1, thereby reducing KAT1 activity, which suggests that active SnRK2.6 negatively regulates KAT1 via phosphorylation to promote stomatal closure $^{[81]}$. Therefore, SnRK2s are important in mediating ABAinduced stomatal closure through the regulation of ion channels.

\section{ABF transcription factors}

The accumulation of ABA in plant cells leads to changes in gene expression that generally contribute to drought stress tolerance. Transcriptome studies in rice and Arabidopsis have shown that exposure to $\mathrm{ABA}$ and various abiotic stresses results in changes to approximately $5 \%-10 \%$ of the genome, with more than half of these changes being common to drought, salinity and ABA treatments ${ }^{[82,83]}$. ABA-induced genes encode proteins involved in stress tolerance and include (i) dehydrins, which are members of the Late Embryogenesis Abundant (LEA) proteins and function as molecular chaperones to stabilize membranes and protect proteins against aggregation; (ii) enzymes that detoxify reactive oxygen species (ROS); and (iii) regulatory proteins such as transcription factors, protein phosphatases and kinases (Figure 1). ABArepressed genes are enriched for those encoding proteins associated with cell growth.

Many cis-acting DNA elements have been identified by analyzing the promoters of ABA-responsive genes ${ }^{[84]}$. Designated as ABA-responsive elements (ABREs), these commonly contain the PyACGTGG/TC consensus sequence belonging to the G-box family (CACGTG), which has been implicated in a wide range of gene expression mechanisms in plants ${ }^{[85]}$. ABAresponsive gene expression requires multiple ABREs or the combination of an ABRE with a coupling element ${ }^{[86,87]}$.

The ABRE-binding (AREB) proteins, or ABRE-binding factors $(\mathrm{ABFs})$, were isolated by using $\mathrm{ABRE}$ sequences as bait in yeast one-hybrid screens ${ }^{[88,89]}$. The AREB/ABFs encode basicdomain leucine zipper (bZIP) transcription factors belonging to the group A subfamily, comprising nine homologs in the Arabidopsis genome that share a highly conserved C-terminal bZIP domain and three additional $\mathrm{N}$-terminal conserved regions designated $\mathrm{C} 1, \mathrm{C} 2$, and $\mathrm{C}^{[90]}$. These nine homologs can be divided into two groups: the ABI5 family (ABI5, EEL, 
DPBF2/AtbZIP67, DPBF4, and AREB3), the members of which are mainly expressed in seeds and are involved in seed development and maturation ${ }^{[49,91-93]}$, and the AREB/ABF family (ABF1, AREB1/ABF2, AREB2/ABF4, and ABF3), the members of which are mainly expressed in vegetative tissues under abiotic stress conditions ${ }^{[88,89,94-96]}$. While ABF1 is strongly induced by cold, but not by osmotic stress ${ }^{[97]}$, AREB1/ABF2, AREB2/ $\mathrm{ABF} 4$, and $\mathrm{ABF} 3$ are induced by dehydration, high salinity and $\mathrm{ABA}$ treatment during vegetative growth ${ }^{[94]}$. The overexpression of these factors enhances drought stress tolerance ${ }^{[94-96]}$, and triple mutants exhibit impaired stress-responsive gene expression ${ }^{[98]}$, indicating that AREB1, AREB2, and ABF3 are master transcription factors responsible for regulating the ABRE-dependent expression of stress-responsive genes.

Several studies suggest that ABA-dependent phosphorylation is required for AREB/ABFs to be full activated. AREB1 requires $A B A$ to achieve full activation, which is regulated by the ABA-dependent multi-site phosphorylation of the conserved domains in AREB1 ${ }^{[99]}$. ABA-activated SnRK2s, including SnRK2.2, SnRK2.3, and SnRK2.6, have been shown to phosphorylate AREB1 ${ }^{[99]}$. Loss of SnRK2.2 and SnRK2.3 function resulted in reduced ABF phosphorylation ${ }^{[71]}$, and ABAinduced gene expression was eliminated in a triple mutant lacking SnRK2.2, SnRK2.3, and SnRK2.6 $6^{[72]}$. These results suggest that SnRK2s are essential for ABA-induced gene expression through the phosphorylation and activation of ABFs. However, the molecular events leading to the ABA-dependent activation of SnRK2s had remained elusive until recent years, and the identification of ABA receptors was an important step that helped bridge the gap in our understanding of $A B A$ perception and SnRK2 activation.

\section{Model of the core ABA signaling pathway}

Based on the aforementioned studies, which independently showed that PYLs bind ABA and inhibit PP2Cs, a model of the core ABA signaling pathway has emerged that links ABA recognition to the transcriptional activation of ABA-responsive genes (Figure 1). In this model, the ABA response is kept silent under basal condition by PP2Cs including ABI1, ABI2 and HAB1, which negatively regulate ABA responses. These phosphatases act by inhibiting SnRK2s including SnRK2.2, SnRK2.3, and SnRK2.6, which are positive regulators of the ABA signaling pathway. High levels of ABA induce PYLs, which are putative ABA receptors, to bind and inhibit PP2Cs. This relieves the PP2C-dependent inhibition of SnRK2s, thereby allowing these kinases to autophosphorylate and activate downstream effectors such as ion channels and ABF transcription factors, which results in the activation of ABA responses.

This model has been elegantly supported by the in vitro reconstitution of the core ABA signaling pathway. Coexpressing a set of the core ABA signaling components, namely, PYLs, PP2Cs, SnRK2s, and ABF2 transcription factors, in plant protoplasts was found to be necessary and sufficient to induce the expression of an ABA-responsive reporter gene upon treatment with $\mathrm{ABA}^{[100]}$. In another study, in vitro reconstitution analyses performed using PYR1, ABI1, and SnRK2 showed that PYR1 inhibited the ABI1-mediated suppression of SnRK2 activity in an ABA-dependent manner ${ }^{[101]}$, which supports the proposed model of the core ABA signaling pathway. As discussed below, this model has provided the framework for further structural and biochemical studies aimed at addressing the precise regulatory mechanisms governing $\mathrm{ABA}$ signaling from ABA perception to SnRK2 activation.

\section{Structural elucidation of the early ABA signaling mechanisms \\ Helix-grip fold receptors}

The first reports identifying PYL family members as candidate ABA receptors were quickly followed by a wave of crystallographic studies, which provided structural evidence supporting the role of this family of proteins as bona fide ABA receptors as well as a mechanistic basis for early ABA signaling events. The earliest PYL structural studies were based on PYR1, PYL1, and PYL2 ${ }^{[102-106]}$. To date, the structures of PYL3, PYL5, PYL9, PYL10, and PYL13 have also been solved, either in their apo or ligand-bound forms or in complexes with PP2 $C^{[107-110]}$. The currently available PYL structures represent more than half of the 14-member family of PYL proteins in Arabidopsis, and all of them exhibit the helix-grip fold, a hallmark of START domain/Bet v 1-fold proteins, which is characterized by a long C-terminal a-helix surrounded by a curved anti-parallel $\beta$-sheet and several smaller a-helices.

\section{Transmitting the ABA signal to PP2Cs}

A distinctive feature of the PYL structure is the occurrence of a large ligand-binding pocket between the C-terminal helix and the central $\beta$-sheet. The entrance of this pocket is guarded by two functionally important $\beta$-loops, which have been termed the 'gate' and 'latch' loops ${ }^{[102]}$ (alternatively named Pro-Cap and Leu-Lock ${ }^{[104]}$, CL2, and CL3 ${ }^{[106]}$, and the $\beta 3-\beta 4$ and $\beta 5-\beta 6$ lid loops $\left.{ }^{[103]}\right)$, that possess the conserved amino acid sequences SGLPA and HRL. The conformations of the gate and latch loops play critical roles in transmitting the ABA binding signal to downstream effectors ${ }^{[102,104-106]}$. Notably, the gate loops of the ligand-free PYR1, PYL1, and PYL2 structures exist in an open conformation that allows ligand entry (Figure 5A, 5B). In contrast, the conformations of these loops are observed to close off the entrance to this pocket in the ABA-bound receptor structures (Figure 5C). ABA binding within the receptor pocket is mediated by a network of polar and non-polar interactions, whereby a conserved lysine residue in the receptor (K59, K86, and K64 in PYR1, PYL1, and PYL2, respectively) anchors the carboxylate group of $\mathrm{ABA}$ to the inner end of the pocket while the cyclohexene ring of $\mathrm{ABA}$ interacts with the receptor gate near the pocket entrance to pull the gate into a closed conformation (Figure 5D-5F). The closure of the gate loop onto the latch loop forms a new gate and latch interface that is necessary for the interaction with PP2C.

The ability to induce receptor gate closure is therefore a key feature of ABA agonist activity, which has been supported by various in vitro and in vivo assays showing that mutations in 

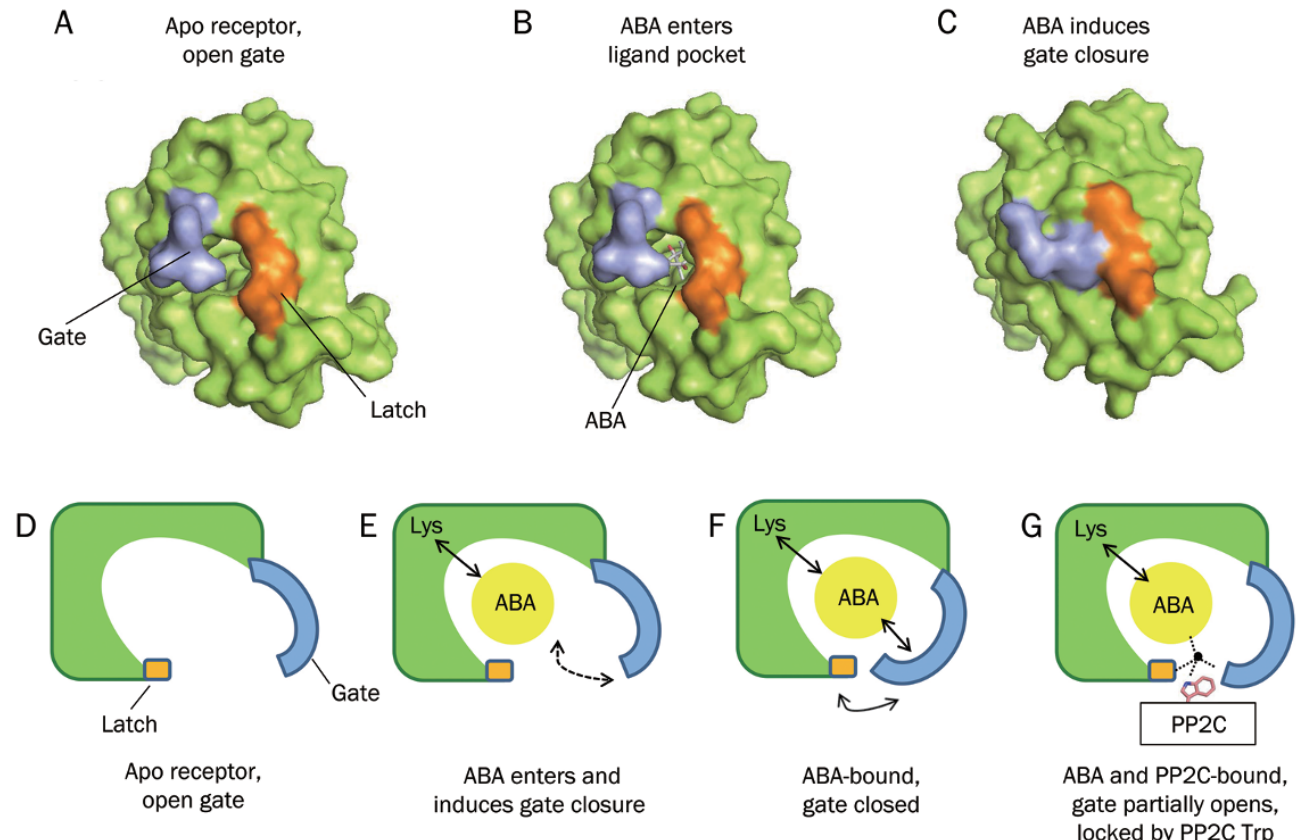

Figure 5. ABA-induced gate closure in PYL. (A) Surface representation of the apo PYL2 (PDB code: 3KAZ) structure with an open ligand binding pocket guarded by the gate (blue) and latch (orange) loops. (B) The same PYL2 structure as in (A), showing ABA entering the pocket. (C) Surface representation of ABA-bound PYL2 (PDB code: $3 \mathrm{KBO}$ ) with a closed pocket. (D-G) Cartoon illustrations of the ligand pocket region of PYL, highlighting the structural mechanisms of the receptor's interaction with ABA and PP2C. The gate and latch loops of the receptor are shown in blue and orange, respectively. (D) The gate loop of the apo receptor is in an open conformation, allowing access to the ligand binding pocket. (E) ABA enters the receptor pocket and binds to a lysine residue via charge interaction. At the outer end of the pocket, ABA interacts with gate residues through hydrophobic interactions. (F) The gate loop closes and forms interactions with the latch residues. (G) In the PYL-ABA-PP2C complex, the receptor gate is partially opened, allowing the insertion of a tryptophan residue from PP2C, which stabilizes the gate and latch interface through a water-mediated interaction network (shown by dashed lines); thus, this residue is termed the "tryptophan lock".

the receptors' gate and latch loops impair their signal transduction abilities ${ }^{[102]}$. Consistent with these findings, structural studies using pyrabactin, a selective ABA agonist, showed that selective receptor activation is related to the closure of the receptor gate. In these studies, pyrabactin-PYL structures in the agonist conformation resemble ABA-PYL structures with the receptor gate in the closed conformation ${ }^{[111-113]}$. Conversely, in pyrabactin-antagonized PYL2 structures, pyrabactin adopts non-productive configurations within the receptor pocket that fail to pull the receptor gate into the active, closed state, thereby explaining its preferentially antagonistic activity $^{[112-114]}$.

Crystal structures of PYL-ABA-PP2C complexes ${ }^{[102,103,106]}$, represented by the PYL2-ABA-HAB1 structure shown in Figure $6 \mathrm{~B}$, revealed that the $\mathrm{ABA}$-bound receptor binds to the active site of $\mathrm{PP} 2 \mathrm{C}$, thereby inhibiting the phosphatase activity both competitively, by blocking the PP2C catalytic site (competition with SnRK2 substrate) $)^{[115]}$, and non-competitively, via hydrogen-bonding of the gate serine hydroxyl group with the catalytic glutamate of PP2C (E203 in HAB1) $)^{[116]}$. The PYL proteins interact with PP2Cs at the gate-latch interface induced by ABA-binding. In this interface, a conserved tryptophan residue in PP2C (W385 in HAB1 and W300 in ABI1) forms a water-mediated contact with $\mathrm{ABA}$ and interacts with the receptor's gate and latch loops, further locking these loops in a closed conformation (Figure 5G). The stabilization of this interface explains the more than 10-fold increase in the ABA-binding affinity of PYL in the presence of PP2 $C^{[4,7,103,106]}$. These structures also explain the previously characterized ABA-insensitive abi1-1 and abi2-1 mutations, in which a glycine-to-aspartic acid change in the PP2C active site disrupts receptor-PP2C interactions ${ }^{[4,6,7,62]}$. In the PYL-ABA-PP2C structures, this glycine is in close proximity to the receptor gate, and the replacement of the glycine hydrogen with the bulky side chain of aspartate sterically interferes with the gate interaction $^{[102,103,106]}$.

\section{Receptor diversity and oligomerization states}

The 14 members of the PYL family share between 38\% and $84 \%$ amino acid sequence identity in the full-length proteins ${ }^{[102]}$. Reconstitution assays in Arabidopsis protoplasts showed that all PYL members except for PYL13, which is ABA-insensitive ${ }^{[110]}$ and was therefore not tested, are able to activate ABA-induced gene expression ${ }^{[100]}$, suggesting functional overlap between the PYL members. In line with this notion, multiple mutations, such as pyr1pyl1pyl2pyl4 quadruple mutations ${ }^{[6]}$ and pyr1pyl1pyl2pyl4pyl5pyl8 sextuple mutations ${ }^{[117]}$, are required to generate robust ABA-insensitive 
A

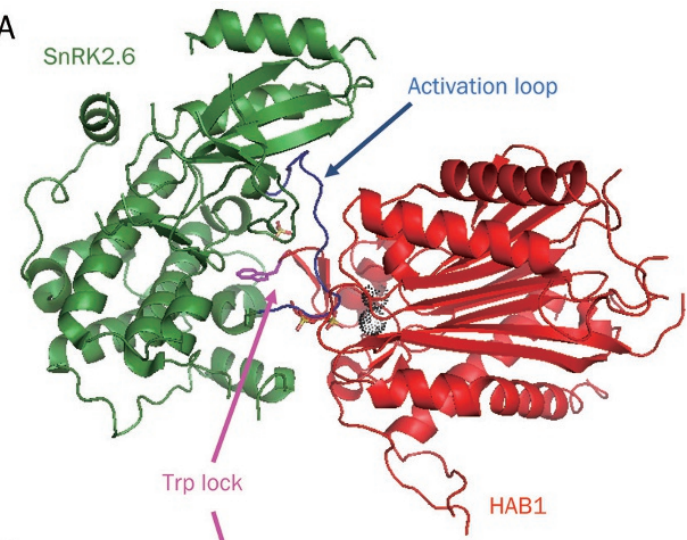

B

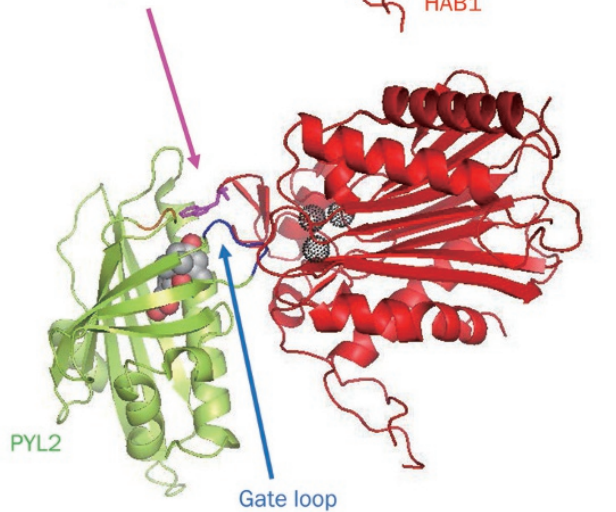

C

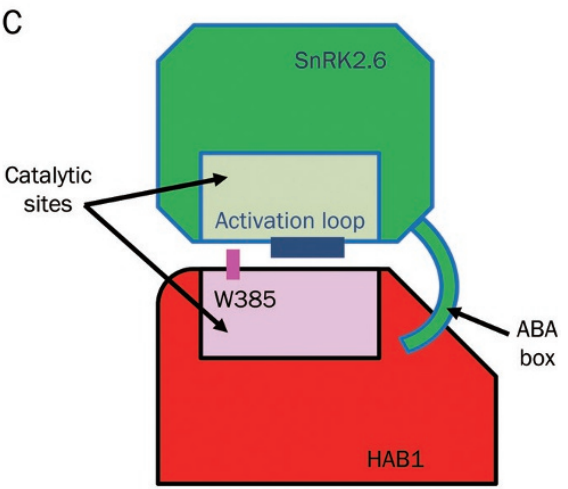

D

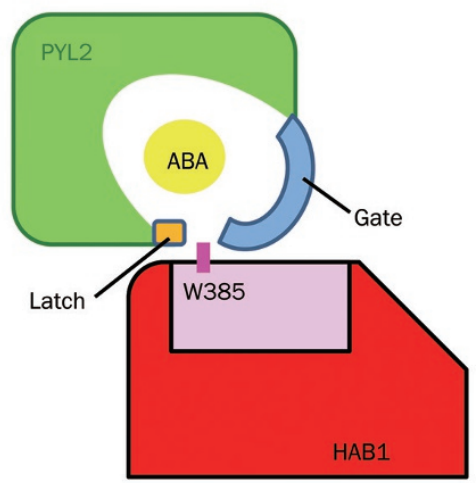

Figure 6. PP2C inhibition of SnRK2. (A) Structure of the SnRK2.6-HAB1 complex (PDB code: 3UJG). SnRK2.6 is shown in dark green, with its activation loop in dark blue. HAB1 is shown in red. The side chain of the conserved PP2C "tryptophan lock" is shown as a stick representation in pink. Magnesium ions and sulfate ions are shown as dot and stick models, respectively. (B) Structure of the PYL2-ABA-HAB1 complex (PDB code: 3KB3) showing the molecular mimicry between PYL2 and SnRK2.6 (above) in their interaction with PP2C. PYL2 is shown in light green, with its gate loop in blue. (C) Cartoon illustration of the two interaction interfaces between SnRK2.6 and HAB1. (D) Cartoon illustration of the PYL2-ABA-HAB1 complex showing that PYL2, as with SnRK2.6 above, interacts with HAB1 at the PP2C catalytic site.

phenotypes. However, other lines of evidence suggest that the functions of different PYL members are not completely redundant. For instance, gene expression studies have revealed both overlapping and differential PYL expression patterns in different tissues ${ }^{[117]}$. Recently, it has been shown that a single pyl8 mutant displayed reduced ABA-mediated inhibition of root growth, indicating a non-redundant role of PYL8 in regulating root sensitivity to $\mathrm{ABA}^{[118]}$.

PYL members have been classified into two groups based on their oligomeric states (Table 1). Using static light scattering and analytical ultracentrifugation, it has been shown that PYL4-10 (except for the untested PYL7) are monomers ${ }^{[107]}$. In contrast, PYR1 and PYL1-3 are largely homodimers in solution in the absence of $\mathrm{ABA}$, but dissociate into monomers in the presence of ABA ${ }^{[107,108,119]}$. The structures of apo PYR1 and

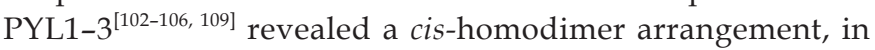
which the two PYL monomers associate in a parallel orientation and thereby block access to their ligand-binding pockets. PYL3 has also been shown to form trans-homodimers that can bind ABA and dissociate into monomers more easily ${ }^{[109]}$, consistent with a mixed monomer/dimer distribution ${ }^{[119]}$.

Because the ligand-binding pocket is blocked in the cishomodimer conformation, and because the gate loops in the open conformation form part of the dimerization interface, ABA binding requires dimer dissociation, resulting in an almost two-orders-of-magnitude lower ABA affinity compared with the monomeric ABA receptors ${ }^{[107,119]}\left(K_{d} \sim 50-100\right.$ $\mu \mathrm{mol} / \mathrm{L}$ for dimeric and $K_{\mathrm{d}} \sim 1 \mu \mathrm{mol} / \mathrm{L}$ for monomeric receptors; Table 1) and a reduced-to-undetectable PP2C affinity in the absence of ABA. Conversely, the monomeric receptors PYL5-10 are able, at sufficient concentrations, to inhibit PP2Cs in the absence of ABA. Moreover, PYL10 has larger hydrophobic residues in the ligand-binding pocket that can partially stabilize gate closure in the apo receptor, which allowed the crystallization of apo PYL10 in both the open ${ }^{[107]}$ and closed gate conformations ${ }^{[120]}$. It is important to note that these interactions are still greatly enhanced by $\mathrm{ABA}^{[4,7,107]}$. For instance, the affinity of PYL10 for the PP2C ABI1 is $1.2 \mu \mathrm{mol} / \mathrm{L}$ in the absence of $\mathrm{ABA}$ and $18 \mathrm{nmol} / \mathrm{L}$ in the presence of $\mathrm{ABA}^{[107]}$. Thus, the monomeric PYLs may function physiologically as highly sensitive ABA receptors rather than as ABAindependent receptors. While the ABA-unresponsive PYL13 does not homodimerize in solution, it is able to heterodimerize with other PYL members and consequently antagonize their activities in the heterodimeric state ${ }^{[121]}$. Notably, PYL10 is incapable of ABA-independent PP2C interactions in the context of 
the PYL10-PYL13 heterodimer complex ${ }^{[110]}$. While it is unclear if PYL receptors do make ABA-independent interactions with PP2Cs under physiological conditions, an understanding the in vitro mechanism of constitutive receptor activation can be applied to the generation of transgenic plants with enhanced ABA sensitivities. The existence of 14 apparently similar PYL proteins with varying properties, such as expression pattern, ABA sensitivity and the extent of PP2C inhibition, therefore provides a complex regulatory system for the fine tuning of the $\mathrm{ABA}$ response.

\section{PP2C regulation of SnRK2}

The crystal structure of a HAB1-SnRK2.6 fusion protein complex, together with biochemical and mutational analyses, revealed a number of interesting features regarding how PP2Cs inhibit SnRK2s in the absence of an ABA signal ${ }^{[115]}$. Two interaction interfaces have been identified, one of which appears in the complex structure as a mutual packing of the phosphatase and kinases active sites. In this interface, the activation loop of the kinase docks into the active site of the PP2C while the conserved ABA-sensing tryptophan of the PP2C inserts into the kinase catalytic cleft (Figure 6A), which resembles the PYL-ABA-PP2C interaction (Figure 6B). This observation revealed a surprising amount of molecular mimicry between the PYL and SnRK2 proteins for occupancy of the same PP2C catalytic site in the PYL-PP2C-SnRK2 signaling cascade.

The second PP2C-SnRK2 interaction interface involves charge interactions between the highly acidic C-terminal ABA box of SnRK2 and a positively charged surface region of PP2C (Figure 6C). This interface, although unresolved in the crystal structure, has been strongly hinted at through extensive biochemical and mutational evidence ${ }^{[115,122]}$. Collectively, these data suggest that the inhibition of SnRK2s by PP2Cs occurs via a two-step mechanism. In the first step, the kinase activity is drastically reduced, but not completely inhibited, by enzymatic dephosphorylation of a critical serine residue in the kinase activation loop, which can occur at low PP2C:SnRK2 molar ratios ${ }^{[115]}$. The second step, involving the full inhibition of kinase activity, is achieved by steric inhibition through the mutual packing of the active sites, as observed in the crystal structure, and requires the formation of stable, stoichiometric PP2C-SnRK2 complexes ${ }^{[115]}$.

\section{SnRK2 activation}

Understanding how PP2C inhibits SnRK2 has provided partial explanations as to how SnRK2 gains activity upon the relief of this inhibition. Analyses of SnRK2 structures in active and inactive conformations provided further insights into the mechanisms of kinase autoactivation. Three independent groups reported the structures of ABA-responsive SnRK2s in late 2011 and early 2012 $2^{[122-124]}$. SnRK2 kinase domains adopt the canonical bilobal kinase fold, with the smaller $\mathrm{N}$-terminal lobe connected to the larger C-terminal lobe by a flexible hinge, and the cleft formed between the two lobes contains the sites for substrate and ATP binding (Figure 7). In all of these structures, the C-terminal regulatory SnRK2 box forms a wellordered a-helix that packs in parallel against the aC helix in the N-terminal lobe, forming extensive contacts that stabilize the position of the aC helix.

The position of the aC helix regulates the closing and opening of the catalytic cleft and thus the alignment of the catalytic residues within the cleft. In many kinases, the position of this regulatory $\mathrm{aC}$ helix is adjusted by activating proteins that bind to the kinase domain ${ }^{[125-131]}$. By analogy, the extensive interactions formed between the crucial SnRK2 box helix and the aC helix stabilize the aC helix, consistent with the requirement for the SnRK2 box and the SnRK2 box - aC helix-interacting
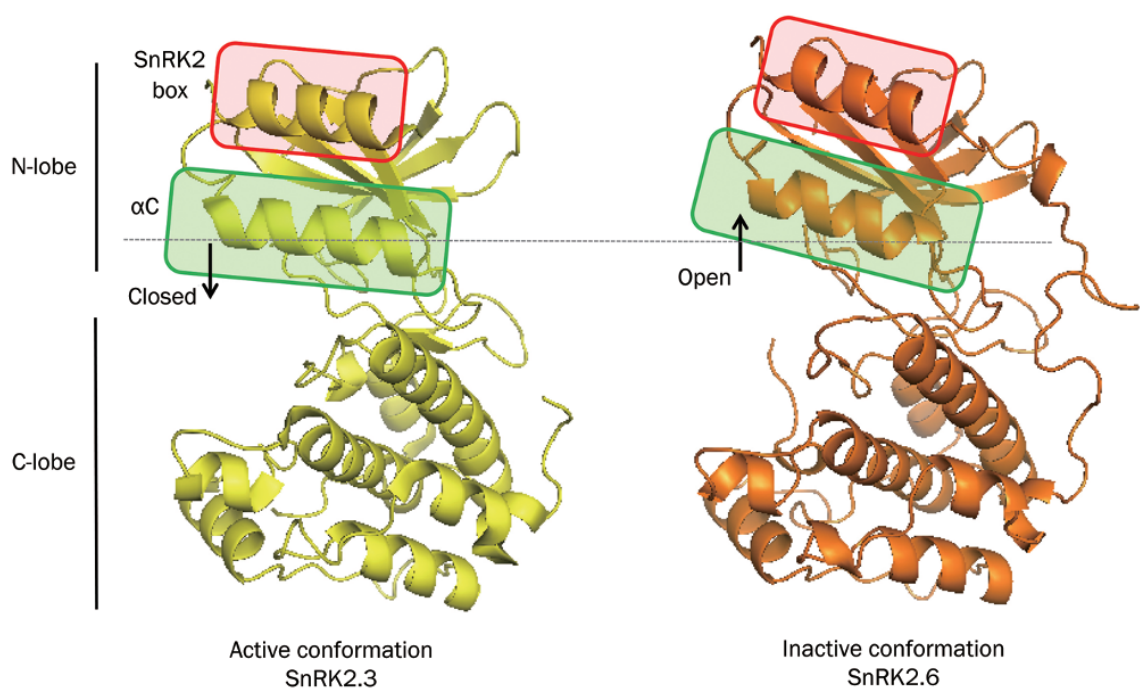

Figure 7. Unphosphorylated SnRK2s can adopt both active and inactive conformations. Structure of SnRK2.3 in the closed conformation (PDB code: 3UC3) in yellow and SnRK2.6 in the open conformation (PDB code: 3UC4) in orange. The SnRK2 box and $\alpha \mathrm{C}$ helices are boxed in red and green, respectively. 
residues for kinase activity.

Although phosphorylation of the activation loop serine is required for full kinase activation, SnRK2s in which this serine is mutated to alanine still retain basal kinase activity ${ }^{[123,132]}$. This basal kinase activity can be explained by the SnRK2 boxstabilized aC helix, which allows unphosphorylated SnRK2s to adopt the conformational characteristics of both inactive and active kinases. This has been illustrated by crystal structures of recombinant unphosphorylated SnRK2s, which adopted either a "closed" conformation (SnRK2.3) and an interaction network characteristic of active kinases or an "open" conformation (SnRK2.6) indicative of inactive kinases (Figure 7) ${ }^{[123]}$. In the "closed" active conformation, the N-terminal lobe is positioned closer to the $\mathrm{C}$-terminal lobe, producing a network of interactions that stabilize $\mathrm{Mg}^{2+}$ and ATP in the catalytic cleft $^{[130,133-135]}$. As SnRK2.2, SnRK2.3, and SnRK2.6 show similar levels of phosphorylation-independent basal activity, it is believed that each of these kinases can spontaneously adopt either an "open" or "closed" conformation ${ }^{[123]}$, suggesting that SnRK2s in the partially active state can align the ATP and $\mathrm{Mg}^{2+}$ molecules for activity.

These results therefore illustrate a two-step activation mechanism of SnRK2 kinases. The first step is the ABA-mediated partial activation of SnRK2 kinase through the release of PP2C-mediated inhibition (Figure 8A-8D). This converts the kinase from a completely inactive state to a partly active state with basal kinase activity due to its ability to spontaneously adopt both "open" and "closed" conformations (Figure 8E). This partial activity allows the kinase to undergo further conversion into the fully active state via activation loop autophosphorylation (Figure 8F), which likely occurs both intra- and intermolecularly ${ }^{[123]}$.

\section{Concluding notes on the collective structural findings}

Altogether, these structural studies highlight the multiple levels of regulation that act upon the key components of the ABA signaling pathway. The inhibition of SnRK2 by PP2C occurs through the enzymatic dephosphorylation of the kinase activation loop serine residue as well as through the physical blockade of the kinase active site, which is accompanied by an allosteric change in the aC helix position. In the relay of the ABA signal, the inhibition of PP2C by ABA-bound PYL involves both the steric blocking of the phosphatase catalytic site and the direct binding of a key catalytic glutamate residue (E203 in HAB1) by the receptor-gate-loop serine residue ${ }^{[116]}$. As a result, the activation of SnRK2 is achieved through the

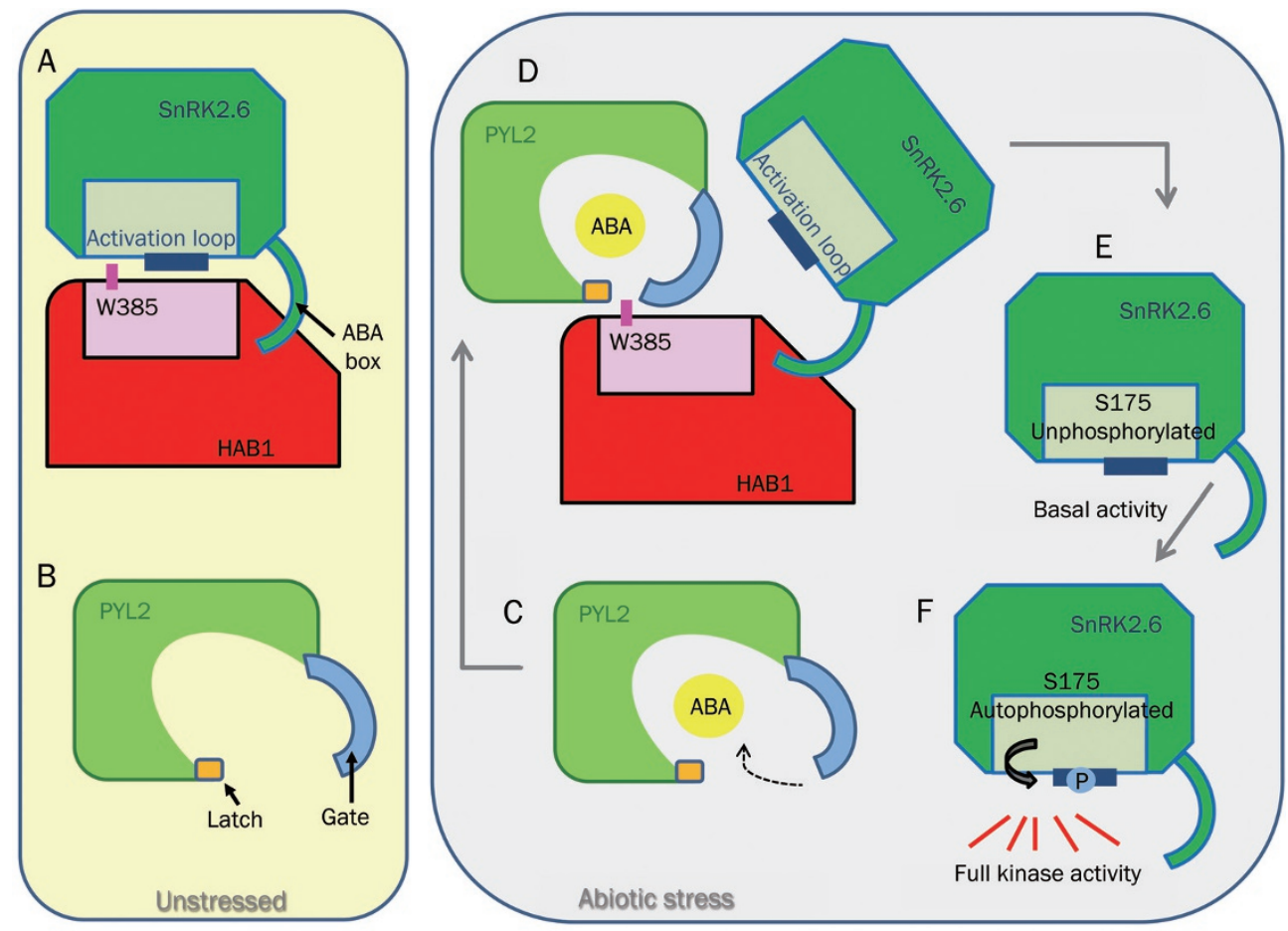

Figure 8. Cartoon summary of the steps leading to SnRK activation. (A) Under unstressed conditions, PP2Cs (represented by HAB1) silence the ABA response by inhibiting SnRK2 kinase activity through a two-step mechanism: first via the enzymatic dephosphorylation of the SnRK2 activation loop serine residue (S175 in SnRK2.6) and second by sterically blocking the kinase catalytic site. HAB1 and SnRK2 are shown in red and dark green, respectively, with their catalytic sites shown in paler shades. The SnRK2.6 activation loop is shown in dark blue. (B) In the absence of ABA, the receptor gate loop is in an open conformation, hindering PP2C binding. (C) The ABA concentration increases under stress conditions, and ABA is sensed by the receptor when hormone binding induces the closure of the receptor gate. (D) ABA-bound PYL2 competes with SnRK2 for PP2C binding. The formation of a stable PYL2-ABA-HAB1 complex inactivates PP2C. (E) SnRK2 is relieved of steric inhibition by PP2C and gains basal activity by spontaneously alternating between active and inactive conformations. (F) Autophosphorylation of the activation loop serine results in full kinase activity. 
inhibition of PP2C catalytic activity as well as through competition for PP2C binding by ABA-bound PYL. Future efforts will likely unravel further levels of complexity in the core signaling pathway as well as in the regulation of the downstream players and furnish a more complete picture of the ABA pathway.

\section{Use of structural information on ABA signaling in agricul- ture \\ Genetic engineering approach}

The understanding of how PYL proteins transmit ABA signals provides opportunities for the development of new tools to improve stress tolerance in plants. One such approach involves the genetic engineering of $\mathrm{ABA}$ receptors to increase their activity. For instance, transgenic plants expressing PYL2 proteins carrying activating mutations that stabilize the receptor-PP2C interactions have shown increased activation of ABA signaling in seeds ${ }^{[136]}$. However, the expression of the constitutively active mutant receptor (PYL2 ${ }^{\mathrm{CA}}$ ) was not detected in vegetative tissues in this study, thereby precluding further analysis in adult plants. In another study, transgenic expression of a PYL4 mutant (PYL4 ${ }^{\mathrm{A} 194 \mathrm{~T}}$ ), which had been selected for its ABA-independent inhibition of PP2CA in vitro, resulted in enhanced drought tolerance in plants ${ }^{[137]}$. Although this study showed promising results, a constitutively active receptor under a strong promoter might affect plant growth under normal conditions, which could lead to decreases in crop yield. Such a drawback could be overcome through the use of inducible promoters to drive receptor expression only under drought conditions. Alternatively, approaches using ABA agonists have also been explored.

\section{Design of new ABA agonists}

The pivotal role of ABA in promoting plant survival under stress conditions has fuelled efforts to use this compound in field trials aimed at improving crop survival. However, the agricultural application of the natural ABA hormone has been largely limited by its short-lived bioactivity, which is due to its instability in solution, susceptibility to photodegradation ${ }^{[138]}$ and rapid catabolism in plants ${ }^{[139]}$. As such, synthetic compounds that functionally mimic ABA but exhibit longer periods of bioactivity are highly sought after. In addition to agricultural uses, ABA agonists may also have commercial value as holding agents to extend the marketing period for plant seedlings and facilitate the transport of ornamental plants ${ }^{[140]}$, further highlighting the importance of identifying a suitable analog capable of mimicking the effects of ABA.

Previously, the rational design of ABA analogs was guided by knowledge of the hormone's chemical structure and catabolic pathway. Chemical libraries of ABA mimetics were generated through the substitution of side groups that are known to be targeted by the plant enzymes involved in ABA catabolism. Compounds such as $3^{\prime}$-fluoro-ABA were designed to resist isomerization into phaseic acid ${ }^{[141]}$, while 8 '-methylene-ABA and 8'-acetylene-ABA irreversibly inhibit ABA 8'-hydroxylase, which catalyzes the predominant step in the breakdown of $\mathrm{ABA}^{[142]}$. However, some of these compounds, such as $8^{\prime}$-trifluoro-ABA, which resists hydroxylation at the C-8 position, are difficult to synthesize and hence impractical for agricultural use ${ }^{[143]}$. Additionally, analogs that target the ABA catabolic pathway run the risk of producing long-lasting effects on the plant that can eventually lead to sustained growth retardation, which is undesirable for agricultural use ${ }^{[140]}$.

The ability of pyrabactin to act as a selective agonist not only played a pivotal role in overcoming the problem of genetic redundancy in the identification of ABA receptors but also enabled an understanding of the fine mechanistic details underlying ABA receptor activation. Although the direct application of pyrabactin in fields is not favorable, given that pyrabactin acts strongly in seeds but not in the vegetative tissues where drought stress tolerance is required, structural studies using pyrabactin have nonetheless provided a valuable framework for the design of novel ABA agonists. Structural analyses of PYL receptors bound to both the pan-agonist ABA and the selective agonist pyrabactin have led to the identification of important interactions within the receptors that are needed to trigger the conformational changes associated with receptor activation ${ }^{[102-106,112]}$. Pyrabactin binds to PYR1 in a manner similar to $\mathrm{ABA}$, with its naphthalene double ring positioned akin to the cyclohexene ring of $\mathrm{ABA}$ and with the bromide group in this ring interacting with the L-P-A residues in the gate loop to help keep it in the closed conformation (Figure 9A, 9B). The sulfonamide group of pyrabactin functionally mimics the carboxylate group of ABA by forming extensive interactions with the receptor, including a water-mediated hydrogen bond with the conserved K86 residue within the ligand-binding pocket. Based on this rationale, Melcher et al conducted a search for $\mathrm{ABA}$ receptor activators containing the naphthalene-1-sulfonamide group using virtual chemical libraries and identified 4 compounds that activated PYR1 in vitro with efficacies comparable to that of pyrabactin ${ }^{[122]}$.

More recently, another ABA analog that also contains a sulfonamide group has been reported by two independent groups to be a potent agonist of several ABA receptors ${ }^{[41,144]}$. This compound has been named AM1 (ABA mimic-1) and quinabactin (for its dihydroquinolinone ring and similar scaffold as pyrabactin) by the two teams (Figure 3). Structural studies carried out by both teams showed that the binding of AM1/quinabactin to PYL2 resembles the binding of ABA more than it does the binding of pyrabactin. The carbonyl group of AM1/quinabactin mimics that of ABA to form a watermediated hydrogen bond with the W385 tryptophan lock from HAB1 in the complex, whereas such a contact is not observed in the pyrabactin-activated receptor-PP2C complexes (Figure 9C, 9D compared with Figure 9A, 9B). The sulfonamide group of AM1/quinabactin, which is in a reverse orientation compared with that of pyrabactin, is positioned at the hydrophilic inner end of the pocket and forms water-mediated or direct hydrogen bonds with receptor residues that also interact with $\mathrm{ABA}$ and pyrabactin. These structural features suggest that AM1/quinabactin elicits $\mathrm{ABA}$ responses through a similar 
A PYL2-ABA-HAB1

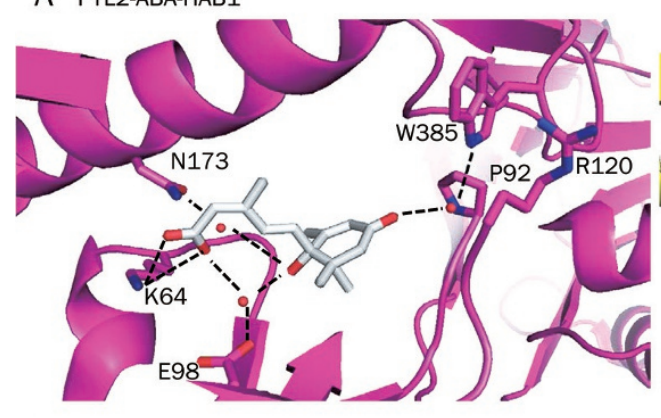

C PYL2-AM1-HAB1 (Cao et al)

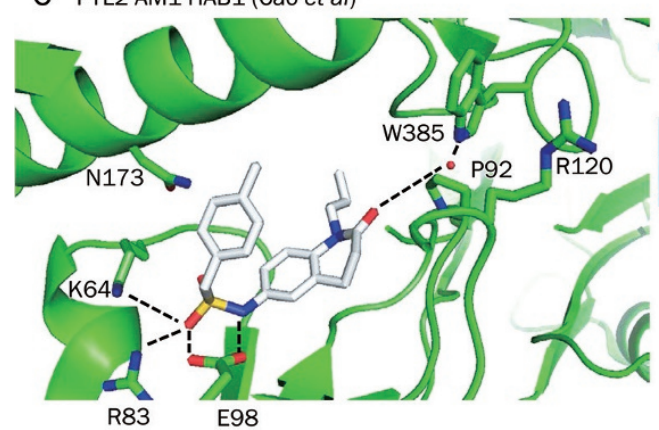

B PYL1-pyrabactin-ABI1

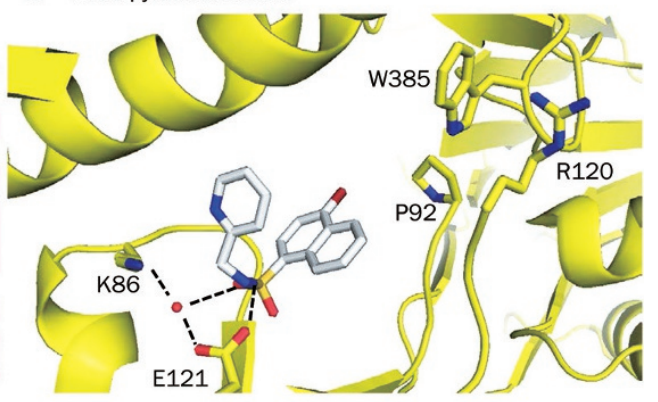

D PYL2-quinabactin-HAB1 (Okamoto et al)

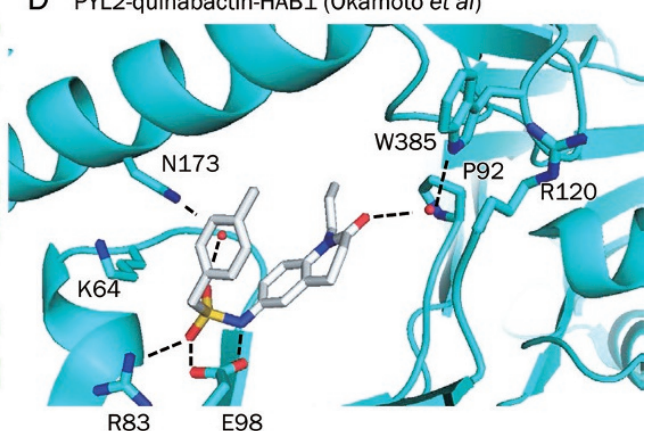

Figure 9. Mode of receptor binding by $A B A$, pyrabactin and AM1/quinabactin. Structures of the ligand-bound pockets of (A) the PYL2-ABA-HAB1 complex (PDB code: 3KB3); (B) the PYL1-pyrabactin-ABI1 complex (3NMN); (C) the PYL2-AMI/Quinabactin-HAB1 complex (PDB code: 4LG5) and (D) the PYL2-AMI/Quinabactin-HAB1 complex (PDB code: 4LA7). Residues that form direct or water-mediated hydrogen bonds (dashed lines) with the ligands are shown in the stick representation. Water-mediated interactions between the ligands' carbonyl oxygen and W385 of HAB1 are shown, which are part of the hydrogen bond network (formed together with P92 and R120 of PYL2, this part of the interaction is not shown) that occurs in the ABA- and AMI/ Quinabactin-bound complexes.

mode of receptor binding as ABA. In vitro, AM1/quinabactin was shown to be more potent than both $\mathrm{ABA}$ and pyrabactin in activating PYR1 and other PYL proteins and in inducing their interactions with HAB1. Although AM1/quinabactin has been shown to preferentially activate dimeric ABA receptors such as PYR1, PYL1, PYL2, and PYL4, AM1/quinabactin was able to produce a similar transcriptional profile as ABA in Arabidopsis. A study of the transcriptomes of seedlings treated with AM1/quinabactin and ABA showed highly correlated activation patterns, with more than $80 \%$ overlap, and the use of a GUS reporter line demonstrated both ABA- and AM1/ quinabactin-triggered transcriptional responses in the guard cells and the vascular tissues of the roots and leaves.

In addition, AM1/quinabactin not only inhibited seed germination to a similar extent as ABA but also increased the drought resistance of plants in the vegetative growth stage. When exogenously applied to wild-type plants subjected to drought conditions, AM1/quinabactin induced reduced water loss, delayed wilting, higher survival rates and better recovery after rehydration compared with non-treated control plants. This compound was also able to rescue the water-loss and wilty phenotype of an ABA-deficient mutant, indicating that AM1/quinabactin functionally mimics $\mathrm{ABA}$ to produce similar physiological responses that ultimately increase drought stress tolerance. However, unlike ABA, which is susceptible to photodegradation, AM1/quinabactin was shown in a pre- liminary study to be more stable with a longer half-life when exposed to mild UV (174 min for AM1 versus 24 min for ABA), making it a more suitable and promising candidate for field applications. It will be necessary to characterize the effects of AM1/quinabactin on crop plants to further substantiate its feasibility for agricultural use.

\section{Concluding remarks}

In view of the effects of global climate change and the population boom on food demand and freshwater resources, there is an impending need to increase agricultural yields while limiting agricultural water consumption. In our constant search for solutions to increase crop production, the ability to improve stress tolerance towards water scarcity and salinity in plants is extremely valuable. In addition, enhancing abiotic stress tolerance to extend shelf-life during the long-distance transport of commercial plants is highly beneficial. Therefore, understanding abiotic stress tolerance in plants has important and widespread implications that cannot be understated.

Our understanding of the ABA pathway has progressed rapidly in the years following the discovery of bona fide ABA receptors and the elucidation of their structures. The application of such structural information for the rational design of $\mathrm{ABA}$ agonists has shown promising signs in the current stages. Although these newly identified compounds require further validation in the field, these studies have demon- 
strated the practical use of applying structural information to providing solutions for current problems. With more effort being directed toward unraveling downstream ABA signaling events and cross-talk with other pathways, the future of the ABA signaling field in plant biology and crop management remains both exciting and promising.

\section{References}

1 Weiner JJ, Peterson FC, Volkman BF, Cutler SR. Structural and functional insights into core ABA signaling. Curr Opin Plant Biol 2010; 13: 495-502.

2 Cutler SR, Rodriguez PL, Finkelstein RR, Abrams SR. Abscisic acid: emergence of a core signaling network. Annu Rev Plant Biol 2010; 61: 651-79.

3 McCourt P, Creelman R. The ABA receptors - we report you decide. Curr Opin Plant Biol 2008; 11: 474-8.

4 Ma Y, Szostkiewicz I, Korte A, Moes D, Yang Y, Christmann A, et al. Regulators of PP2C phosphatase activity function as abscisic acid sensors. Science 2009; 324: 1064-8.

5 Nishimura N, Sarkeshik A, Nito K, Park SY, Wang A, Carvalho PC, et al. PYR/PYL/RCAR family members are major in-vivo ABI1 protein phosphatase 2C-interacting proteins in Arabidopsis. Plant J 2010; 61: 290-9.

6 Park SY, Fung P, Nishimura N, Jensen DR, Fujii H, Zhao Y, et al. Abscisic acid inhibits type $2 \mathrm{C}$ protein phosphatases via the PYR/PYL family of START proteins. Science 2009; 324: 1068-71.

7 Santiago J, Rodrigues A, Saez A, Rubio S, Antoni R, Dupeux F, et al. Modulation of drought resistance by the abscisic acid receptor PYL5 through inhibition of clade A PP2Cs. Plant J 2009; 60: 575-88.

8 Breakthrough of the year. The runners-up. Science 2009; 326: 1600-7.

9 Adler EM. 2009: signaling breakthroughs of the year. Sci Signal 2010; 3: eg1.

10 Zhu JK. Salt and drought stress signal transduction in plants. Annu Rev Plant Biol 2002; 53: 247-73.

11 Saez A, Robert N, Maktabi MH, Schroeder Jl, Serrano R, Rodriguez PL. Enhancement of abscisic acid sensitivity and reduction of water consumption in Arabidopsis by combined inactivation of the protein phosphatases type 2C ABI1 and HAB1. Plant Physiol 2006; 141: 1389-99.

$12 \mathrm{Kim}$ TH, Bohmer M, Hu H, Nishimura N, Schroeder Jl. Guard cell signal transduction network: advances in understanding abscisic acid, $\mathrm{CO}_{2}$, and $\mathrm{Ca}^{2+}$ signaling. Annu Rev Plant Biol 2010; 61: 56191.

13 Xiong L, Ishitani M, Lee H, Zhu JK. The Arabidopsis LOS5/ABA3 locus encodes a molybdenum cofactor sulfurase and modulates cold stress- and osmotic stress-responsive gene expression. Plant Cell 2001; 13: 2063-83.

14 Milborrow BV. The chemistry and physiology of abscisic acid. Annu Rev Plant Physiol 1974; 25: 259-307.

15 Lopez-Molina L, Mongrand S, Chua NH. A postgermination developmental arrest checkpoint is mediated by abscisic acid and requires the ABI5 transcription factor in Arabidopsis. Proc Natl Acad Sci U S A 2001; 98: 4782-7.

16 Xiong L, Schumaker KS, Zhu JK. Cell signaling during cold, drought, and salt stress. Plant Cell 2002; 14: S165-83.

17 Nambara E, Marion-Poll A. Abscisic acid biosynthesis and catabolism. Annu Rev Plant Biol 2005; 56: 165-85.

18 Kuromori T, Miyaji T, Yabuuchi H, Shimizu H, Sugimoto E, Kamiya A, et al. ABC transporter AtABCG25 is involved in abscisic acid transport and responses. Proc Natl Acad Sci U S A 2010; 107: 2361-6.
19 Kang J, Hwang JU, Lee M, Kim YY, Assmann SM, Martinoia E, et al. PDR-type $A B C$ transporter mediates cellular uptake of the phytohormone abscisic acid. Proc Natl Acad Sci U S A 2010; 107: 2355-60.

20 Umezawa T, Nakashima K, Miyakawa T, Kuromori T, Tanokura M, Shinozaki $\mathrm{K}$, et al. Molecular basis of the core regulatory network in ABA responses: sensing, signaling and transport. Plant Cell Physiol 2010; 51: 1821-39.

21 Kanno Y, Hanada A, Chiba Y, Ichikawa T, Nakazawa M, Matsui M, et al. Identification of an abscisic acid transporter by functional screening using the receptor complex as a sensor. Proc Natl Acad Sci U S A 2012; 109: 9653-8.

22 Cutler AJ, Krochko JE. Formation and breakdown of ABA. Trends Plant Sci 1999; 4: 472-8.

23 Okamoto M, Tanaka Y, Abrams SR, Kamiya Y, Seki M, Nambara E. High humidity induces abscisic acid 8'-hydroxylase in stomata and vasculature to regulate local and systemic abscisic acid responses in Arabidopsis. Plant Physiol 2009; 149: 825-34.

24 Zou J, Abrams GD, Barton DL, Taylor DC, Pomeroy MK, Abrams SR. Induction of lipid and oleosin biosynthesis by (+)-abscisic acid and its metabolites in microspore-derived embryos of brassica napus L.cv reston (biological responses in the presence of $8^{\prime}$-hydroxyabscisic acid). Plant Physiol 1995; 108: 563-71.

25 Kushiro T, Okamoto M, Nakabayashi K, Yamagishi K, Kitamura S, Asami T, et al. The Arabidopsis cytochrome P450 CYP707A encodes ABA 8'-hydroxylases: key enzymes in ABA catabolism. EMBO J 2004; 23: $1647-56$.

26 Saito S, Hirai N, Matsumoto C, Ohigashi H, Ohta D, Sakata K, et al. Arabidopsis CYP707As encode (+)-abscisic acid 8'-hydroxylase, a key enzyme in the oxidative catabolism of abscisic acid. Plant Physiol 2004; 134: 1439-49.

27 Umezawa T, Okamoto M, Kushiro T, Nambara E, Oono Y, Seki M, et al. CYP707A3, a major ABA 8'-hydroxylase involved in dehydration and rehydration response in Arabidopsis thaliana. Plant J 2006; 46: 171-82.

28 Dietz KJ, Sauter A, Wichert K, Messdaghi D, Hartung W. Extracellular beta-glucosidase activity in barley involved in the hydrolysis of ABA glucose conjugate in leaves. J Exp Bot 2000; 51: 937-44.

29 Boursiac Y, Leran S, Corratge-Faillie C, Gojon A, Krouk G, Lacombe B. ABA transport and transporters. Trends Plant Sci 2013; 18: 325-33.

30 Burla B, Pfrunder S, Nagy R, Francisco RM, Lee Y, Martinoia E. Vacuolar transport of abscisic acid glucosyl ester is mediated by ATPbinding cassette and proton-antiport mechanisms in Arabidopsis thaliana. Plant Physiol 2013; 163: 1446-58.

31 Lee KH, Piao HL, Kim HY, Choi SM, Jiang F, Hartung W, et al. Activation of glucosidase via stress-induced polymerization rapidly increases active pools of abscisic acid. Cell 2006; 126: 1109-20.

$32 \mathrm{Xu} Z \mathrm{ZY}$, Lee KH, Dong T, Jeong JC, Jin JB, Kanno Y, et al. A vacuolar beta-glucosidase homolog that possesses glucose-conjugated abscisic acid hydrolyzing activity plays an important role in osmotic stress responses in Arabidopsis. Plant Cell 2012; 24: 2184-99.

33 Walker-Simmons MK, Rose PA, Shaw AC, Abrams SR. The 7[prime]methyl group of abscisic acid is critical for biological activity in wheat embryo germination. Plant Physiol 1994; 106: 1279-84.

34 Lin BL, Wang HJ, Wang JS, Zaharia LI, Abrams SR. Abscisic acid regulation of heterophylly in Marsilea quadrifolia L.: effects of R-(-) and S-(+) isomers. J Exp Bot 2005; 56: 2935-48.

35 Huang D, Jaradat MR, Wu W, Ambrose SJ, Ross AR, Abrams SR, et al. Structural analogs of $A B A$ reveal novel features of $A B A$ perception and signaling in Arabidopsis. Plant J 2007; 50: 414-28.

36 Asami T, Robertson M, Yamamoto S, Yoneyama K, Takeuchi Y, Yoshida S. Biological activities of an abscisic acid analog in barley, 
cress, and rice. Plant Cell Physiol 1998; 39: 342-8.

37 Wilen RW, Hays DB, Mandel RM, Abrams SR, Moloney MM. Competitive inhibition of abscisic acid-regulated gene expression by stereoisomeric acetylenic analogs of abscisic acid. Plant Physiol 1993; 101: 469-76.

38 Yamazaki D, Yoshida S, Asami T, Kuchitsu K. Visualization of abscisic acid-perception sites on the plasma membrane of stomatal guard cells. Plant J 2003; 35: 129-39.

39 Nishimura N, Yoshida T, Murayama M, Asami T, Shinozaki K, Hirayama T. Isolation and characterization of novel mutants affecting the abscisic acid sensitivity of Arabidopsis germination and seedling growth. Plant Cell Physiol 2004; 45: 1485-99.

40 Cao M, Liu X, Zhang Y, Xue X, Zhou XE, Melcher K, et al. An ABAmimicking ligand that reduces water loss and promotes drought resistance in plants. Cell Res 2013; 23: 1043-54.

41 Okamoto M, Peterson FC, Defries A, Park SY, Endo A, Nambara E, et al. Activation of dimeric ABA receptors elicits guard cell closure, ABAregulated gene expression, and drought tolerance. Proc Natl Acad Sci U S A 2013; 110: 12132-7.

42 Koornneef M, Reuling G, Karssen CM. The isolation and characterization of abscisic acid-insensitive mutants of Arabidopsis thaliana. Physiol Plant 1984; 61: 377-83.

43 Finkelstein RR. Mutations at two new Arabidopsis ABA response loci are similar to the abi3 mutations. Plant J 1994; 5: 765-71.

44 Meyer K, Leube MP, Grill E. A protein phosphatase 2C involved in ABA signal transduction in Arabidopsis thaliana. Science 1994; 264 : 1452-5.

45 Leung J, Bouvier-Durand M, Morris PC, Guerrier D, Chefdor F, Giraudat J. Arabidopsis ABA response gene ABI1: features of a calcium-modulated protein phosphatase. Science 1994; 264: 1448-52.

46 Leung J, Merlot S, Giraudat J. The Arabidopsis ABSCISIC ACIDINSENSITIVE2 $(A B I 2)$ and $A B I 1$ genes encode homologous protein phosphatases $2 \mathrm{C}$ involved in abscisic acid signal transduction. Plant Cell 1997; 9: 759-71.

47 Parcy F, Valon C, Raynal M, Gaubier-Comella P, Delseny M, Giraudat J. Regulation of gene expression programs during Arabidopsis seed development: roles of the $\mathrm{ABI} 3$ locus and of endogenous abscisic acid. Plant Cell 1994; 6: 1567-82.

48 Finkelstein RR, Wang ML, Lynch TJ, Rao S, Goodman HM. The Arabidopsis abscisic acid response locus ABI4 encodes an APETALA 2 domain protein. Plant Cell 1998; 10: 1043-54.

49 Finkelstein RR, Lynch TJ. The Arabidopsis abscisic acid response gene ABI5 encodes a basic leucine zipper transcription factor. Plant Cell 2000; 12: 599-609.

50 Razem FA, El-Kereamy A, Abrams SR, Hill RD. The RNA-binding protein FCA is an abscisic acid receptor. Nature 2006; 439: 290-4.

51 Shen YY, Wang XF, Wu FQ, Du SY, Cao Z, Shang Y, et al. The Mgchelatase $\mathrm{H}$ subunit is an abscisic acid receptor. Nature 2006; 443: 823-6.

52 Liu X, Yue Y, Li B, Nie Y, Li W, Wu WH, et al. A G protein-coupled receptor is a plasma membrane receptor for the plant hormone abscisic acid. Science 2007; 315: 1712-6.

53 Pandey S, Nelson DC, Assmann SM. Two novel GPCR-type G proteins are abscisic acid receptors in Arabidopsis. Cell 2009; 136: 136-48.

54 Schweighofer A, Hirt H, Meskiene I. Plant PP2C phosphatases: emerging functions in stress signaling. Trends Plant Sci 2004; 9: 236-43.

55 Rodriguez PL, Benning G, Grill E. ABI2, a second protein phosphatase $2 \mathrm{C}$ involved in abscisic acid signal transduction in Arabidopsis. FEBS Lett 1998; 421: 185-90.

56 Rodriguez PL, Leube MP, Grill E. Molecular cloning in Arabidopsis thaliana of a new protein phosphatase 2C (PP2C) with homology to ABI1 and ABI2. Plant Mol Biol 1998; 38: 879-83.

57 Robert N, Merlot S, N'Guyen V, Boisson-Dernier A, Schroeder JI. A hypermorphic mutation in the protein phosphatase 2C HAB1 strongly affects ABA signaling in Arabidopsis. FEBS Lett 2006; 580: 4691-6.

58 Saez A, Apostolova N, Gonzalez-Guzman M, Gonzalez-Garcia MP, Nicolas C, Lorenzo O, et al. Gain-of-function and loss-of-function phenotypes of the protein phosphatase $2 \mathrm{C}$ HAB1 reveal its role as a negative regulator of abscisic acid signalling. Plant J 2004; 37 : 354-69.

59 Merlot S, Gosti F, Guerrier D, Vavasseur A, Giraudat J. The ABI1 and $A B I 2$ protein phosphatases $2 \mathrm{C}$ act in a negative feedback regulatory loop of the abscisic acid signalling pathway. Plant J 2001; 25: 295303.

60 Gosti F, Beaudoin N, Serizet C, Webb AA, Vartanian N, Giraudat J. $\mathrm{ABI} 1$ protein phosphatase $2 \mathrm{C}$ is a negative regulator of abscisic acid signaling. Plant Cell 1999; 11: 1897-910.

61 Rubio S, Rodrigues A, Saez A, Dizon MB, Galle A, Kim TH, et al. Triple loss of function of protein phosphatases type $2 \mathrm{C}$ leads to partial constitutive response to endogenous abscisic acid. Plant Physiol 2009; 150: 1345-55.

62 Sheen J. Mutational analysis of protein phosphatase $2 \mathrm{C}$ involved in abscisic acid signal transduction in higher plants. Proc Natl Acad Sci U S A 1998; 95: 975-80.

63 Moes D, Himmelbach A, Korte A, Haberer G, Grill E. Nuclear localization of the mutant protein phosphatase abi1 is required for insensitivity towards ABA responses in Arabidopsis. Plant J 2008; 54: 806-19.

64 Mustilli AC, Merlot S, Vavasseur A, Fenzi F, Giraudat J. Arabidopsis OST1 protein kinase mediates the regulation of stomatal aperture by abscisic acid and acts upstream of reactive oxygen species production. Plant Cell 2002; 14: 3089-99.

65 Yoshida R, Hobo T, Ichimura K, Mizoguchi T, Takahashi F, Aronso J, et al. ABA-activated SnRK2 protein kinase is required for dehydration stress signaling in Arabidopsis. Plant Cell Physiol 2002; 43: 147383.

66 Hrabak EM, Chan CW, Gribskov M, Harper JF, Choi JH, Halford N, et al. The Arabidopsis CDPK-SnRK superfamily of protein kinases. Plant Physiol 2003; 132: 666-80.

67 Polge C, Thomas M. SNF1/AMPK/SnRK1 kinases, global regulators at the heart of energy control? Trends Plant Sci 2007; 12: 20-8.

68 Coello P, Hey SJ, Halford NG. The sucrose non-fermenting-1-related (SnRK) family of protein kinases: potential for manipulation to improve stress tolerance and increase yield. J Exp Bot 2011; 62: 883-93.

69 Boudsocq M, Barbier-Brygoo H, Lauriere C. Identification of nine sucrose nonfermenting 1-related protein kinases 2 activated by hyperosmotic and saline stresses in Arabidopsis thaliana. J Biol Chem 2004; 279: 41758-66.

70 Fujii $\mathrm{H}$, Verslues PE, Zhu JK. Arabidopsis decuple mutant reveals the importance of SnRK2 kinases in osmotic stress responses in vivo. Proc Natl Acad Sci U S A 2011; 108: 1717-22.

71 Fujii $\mathrm{H}$, Verslues PE, Zhu JK. Identification of two protein kinases required for abscisic acid regulation of seed germination, root growth, and gene expression in Arabidopsis. Plant Cell 2007; 19: 485-94.

72 Fujii H, Zhu JK. Arabidopsis mutant deficient in 3 abscisic acidactivated protein kinases reveals critical roles in growth, reproduction, and stress. Proc Natl Acad Sci U S A 2009; 106: 8380-5.

73 Fujita Y, Nakashima K, Yoshida T, Katagiri T, Kidokoro S, Kanamori N, et al. Three SnRK2 protein kinases are the main positive regulators of abscisic acid signaling in response to water stress in Arabidopsis. Plant Cell Physiol 2009; 50: 2123-32. 
74 Nakashima K, Fujita Y, Kanamori N, Katagiri T, Umezawa T, Kidokoro $\mathrm{S}$, et al. Three Arabidopsis SnRK2 protein kinases, SRK2D/ SnRK2.2, SRK2E/SnRK2.6/OST1 and SRK2I/SnRK2.3, involved in ABA signaling are essential for the control of seed development and dormancy. Plant Cell Physiol 2009; 50: 1345-63.

75 Belin C, de Franco PO, Bourbousse C, Chaignepain S, Schmitter JM, Vavasseur A, et al. Identification of features regulating OST1 kinase activity and OST1 function in guard cells. Plant Physiol 2006; 141: 1316-27.

76 Yoshida R, Umezawa T, Mizoguchi T, Takahashi S, Takahashi F, Shinozaki K. The regulatory domain of SRK2E/OST1/SnRK2.6 interacts with $\mathrm{ABI} 1$ and integrates abscisic acid (ABA) and osmotic stress signals controlling stomatal closure in Arabidopsis. J Biol Chem 2006; 281: 5310-8.

77 Vahisalu T, Kollist H, Wang YF, Nishimura N, Chan WY, Valerio G, et al. SLAC1 is required for plant guard cell S-type anion channel function in stomatal signalling. Nature 2008; 452: 487-91.

78 Negi J, Matsuda O, Nagasawa T, Oba Y, Takahashi H, Kawai-Yamada $\mathrm{M}$, et al. CO2 regulator SLAC1 and its homologues are essential for anion homeostasis in plant cells. Nature 2008; 452: 483-6.

79 Geiger D, Scherzer S, Mumm P, Stange A, Marten I, Bauer H, et al. Activity of guard cell anion channel SLAC1 is controlled by droughtstress signaling kinase-phosphatase pair. Proc Natl Acad Sci U S A 2009; 106: 21425-30.

80 Lee SC, Lan W, Buchanan BB, Luan S. A protein kinase-phosphatase pair interacts with an ion channel to regulate $A B A$ signaling in plant guard cells. Proc Natl Acad Sci U S A 2009; 106: 21419-24.

81 Sato A, Sato Y, Fukao Y, Fujiwara M, Umezawa T, Shinozaki K, et al. Threonine at position 306 of the KAT1 potassium channel is essential for channel activity and is a target site for ABA-activated SnRK2/OST1/SnRK2.6 protein kinase. Biochem J 2009; 424: 43948.

82 Nakashima K, Ito Y, Yamaguchi-Shinozaki K. Transcriptional regulatory networks in response to abiotic stresses in Arabidopsis and grasses. Plant Physiol 2009; 149: 88-95.

83 Shinozaki K, Yamaguchi-Shinozaki K, Seki M. Regulatory network of gene expression in the drought and cold stress responses. Curr Opin Plant Biol 2003; 6: 410-7.

84 Busk PK, Pages M. Regulation of abscisic acid-induced transcription. Plant Mol Biol 1998; 37: 425-35.

85 Menkens AE, Schindler U, Cashmore AR. The G-box: a ubiquitous regulatory DNA element in plants bound by the GBF family of bZIP proteins. Trends Biochem Sci 1995; 20: 506-10.

86 Zhang W, Ruan J, Ho TH, You Y, Yu T, Quatrano RS. Cis-regulatory element based targeted gene finding: genome-wide identification of abscisic acid- and abiotic stress-responsive genes in Arabidopsis thaliana. Bioinformatics 2005; 21: 3074-81.

87 Gomez-Porras JL, Riano-Pachon DM, Dreyer I, Mayer JE, MuellerRoeber B. Genome-wide analysis of ABA-responsive elements ABRE and $\mathrm{CE} 3$ reveals divergent patterns in Arabidopsis and rice. BMC Genomics 2007; 8: 260.

88 Choi H, Hong J, Ha J, Kang J, Kim SY. ABFs, a family of ABAresponsive element binding factors. J Biol Chem 2000; 275: 172330.

89 Uno Y, Furihata T, Abe H, Yoshida R, Shinozaki K, YamaguchiShinozaki K. Arabidopsis basic leucine zipper transcription factors involved in an abscisic acid-dependent signal transduction pathway under drought and high-salinity conditions. Proc Natl Acad Sci U S A 2000; 97: 11632-7.

90 Jakoby M, Weisshaar B, Droge-Laser W, Vicente-Carbajosa J, Tiedemann J, Kroj T, et al. bZIP transcription factors in Arabidopsis. Trends Plant Sci 2002; 7: 106-11.
91 Bensmihen S, Giraudat J, Parcy F. Characterization of three homologous basic leucine zipper transcription factors (bZIP) of the ABI5 family during Arabidopsis thaliana embryo maturation. J Exp Bot 2005; 56: 597-603.

92 Bensmihen S, Rippa S, Lambert G, Jublot D, Pautot V, Granier F, et al. The homologous $\mathrm{ABI} 5$ and EEL transcription factors function antagonistically to fine-tune gene expression during late embryogenesis. Plant Cell 2002; 14: 1391-403.

93 Kim SY, Ma J, Perret P, Li Z, Thomas TL. Arabidopsis ABI5 subfamily members have distinct DNA-binding and transcriptional activities. Plant Physiol 2002; 130: 688-97.

94 Fujita Y, Fujita M, Satoh R, Maruyama K, Parvez MM, Seki M, et al. AREB1 is a transcription activator of novel ABRE-dependent ABA signaling that enhances drought stress tolerance in Arabidopsis. Plant Cell 2005; 17: 3470-88.

95 Kang JY, Choi HI, Im MY, Kim SY. Arabidopsis basic leucine zipper proteins that mediate stress-responsive abscisic acid signaling. Plant Cell 2002; 14: 343-57.

96 Kim S, Kang JY, Cho DI, Park JH, Kim SY. ABF2, an ABRE-binding bZIP factor, is an essential component of glucose signaling and its overexpression affects multiple stress tolerance. Plant J 2004; 40: 75-87.

97 Kim SY. The role of ABF family bZIP class transcription factors in stress response. Physiologia Plantarum 2006; 126: 519-27.

98 Yoshida T, Fujita Y, Sayama H, Kidokoro S, Maruyama K, Mizoi J, et al. AREB1, AREB2, and ABF3 are master transcription factors that cooperatively regulate $A B R E-d e p e n d e n t A B A$ signaling involved in drought stress tolerance and require $A B A$ for full activation. Plant $J$ 2010; 61: 672-85.

99 Furihata T, Maruyama K, Fujita Y, Umezawa T, Yoshida R, Shinozaki $\mathrm{K}$, et al. Abscisic acid-dependent multisite phosphorylation regulates the activity of a transcription activator AREB1. Proc Natl Acad Sci U S A 2006; 103: 1988-93.

100 Fujii H, Chinnusamy V, Rodrigues A, Rubio S, Antoni R, Park SY, et al. In vitro reconstitution of an abscisic acid signalling pathway. Nature 2009; 462: 660-4.

101 Umezawa T, Sugiyama N, Mizoguchi M, Hayashi S, Myouga F, Yamaguchi-Shinozaki $\mathrm{K}$, et al. Type $2 \mathrm{C}$ protein phosphatases directly regulate abscisic acid-activated protein kinases in Arabidopsis. Proc Natl Acad Sci U S A 2009; 106: 17588-93.

102 Melcher K, Ng LM, Zhou XE, Soon FF, Xu Y, Suino-Powell KM, et al. A gate-latch-lock mechanism for hormone signalling by abscisic acid receptors. Nature 2009; 462: 602-8.

103 Miyazono K, Miyakawa T, Sawano Y, Kubota K, Kang HJ, Asano A, et al. Structural basis of abscisic acid signalling. Nature 2009; 462: 609-14.

104 Nishimura N, Hitomi K, Arvai AS, Rambo RP, Hitomi C, Cutler SR, et al. Structural mechanism of abscisic acid binding and signaling by dimeric PYR1. Science 2009; 326: 1373-9.

105 Santiago J, Dupeux F, Round A, Antoni R, Park SY, Jamin M, et al. The abscisic acid receptor PYR1 in complex with abscisic acid. Nature 2009; 462: 665-8.

106 Yin P, Fan H, Hao Q, Yuan X, Wu D, Pang Y, et al. Structural insights into the mechanism of abscisic acid signaling by PYL proteins. Nat Struct Mol Biol 2009; 16: 1230-6.

107 Hao Q, Yin P, Li W, Wang L, Yan C, Lin Z, et al. The molecular basis of ABA-independent inhibition of PP2Cs by a subclass of PYL proteins. Mol Cell 2011; 42: 662-72.

108 Zhang X, Jiang L, Wang G, Yu L, Zhang Q, Xin Q, et al. Structural insights into the abscisic acid stereospecificity by the ABA receptors PYR/PYL/RCAR. PLoS One 2013; 8: e67477.

109 Zhang X, Zhang Q, Xin Q, Yu L, Wang Z, Wu W, et al. Complex 
structures of the abscisic acid receptor PYL3/RCAR13 reveal a unique regulatory mechanism. Structure 2012; 20: 780-90.

110 Li W, Wang L, Sheng X, Yan C, Zhou R, Hang J, et al. Molecular basis for the selective and ABA-independent inhibition of PP2CA by PYL13. Cell Res 2013; 23: 1369-79.

111 Hao Q, Yin P, Yan C, Yuan X, Li W, Zhang Z, et al. Functional mechanism of the abscisic acid agonist pyrabactin. J Biol Chem 2010; 285: 28946-52.

112 Melcher K, Xu Y, Ng LM, Zhou XE, Soon FF, Chinnusamy V, et al. Identification and mechanism of ABA receptor antagonism. Nat Struct Mol Biol 2010; 17: 1102-8.

113 Peterson FC, Burgie ES, Park SY, Jensen DR, Weiner JJ, Bingman CA, et al. Structural basis for selective activation of ABA receptors. Nat Struct Mol Biol 2010; 17: 1109-13.

114 Yuan X, Yin P, Hao Q, Yan C, Wang J, Yan N. Single amino acid alteration between valine and isoleucine determines the distinct pyrabactin selectivity by PYL1 and PYL2. J Biol Chem 2010; 285: 28953-8.

115 Soon FF, Ng LM, Zhou XE, West GM, Kovach A, Tan MH, et al. Molecular mimicry regulates ABA signaling by SnRK2 kinases and PP2C phosphatases. Science 2012; 335: 85-8.

116 Zhou XE, Soon FF, Ng LM, Kovach A, Suino-Powell KM, Li J, et al. Catalytic mechanism and kinase interactions of ABA-signaling PP2C phosphatases. Plant Signal Behav 2012; 7: 581-8.

117 Gonzalez-Guzman M, Pizzio GA, Antoni R, Vera-Sirera F, Merilo E, Bassel GW, et al. Arabidopsis PYR/PYL/RCAR receptors play a major role in quantitative regulation of stomatal aperture and transcriptional response to abscisic acid. Plant Cell 2012; 24: 2483-96.

118 Antoni R, Gonzalez-Guzman M, Rodriguez L, Peirats-Llobet M, Pizzio GA, Fernandez MA, et al. PYRABACTIN RESISTANCE1-LIKE8 plays an important role for the regulation of abscisic acid signaling in root. Plant Physiol 2013; 161: 931-41.

119 Dupeux F, Santiago J, Betz K, Twycross J, Park SY, Rodriguez L, et al. A thermodynamic switch modulates abscisic acid receptor sensitivity. EMBO J 2011; 30: 4171-84.

120 Sun D, Wang H, Wu M, Zang J, Wu F, Tian C. Crystal structures of the Arabidopsis thaliana abscisic acid receptor PYL10 and its complex with abscisic acid. Biochem Biophys Res Commun 2012; 418: $122-$ 7.

121 Zhao Y, Chan Z, Xing L, Liu X, Hou YJ, Chinnusamy V, et al. The unique mode of action of a divergent member of the ABA-receptor protein family in ABA and stress signaling. Cell Res 2013; 23: 138095.

122 Xie T, Ren R, Zhang YY, Pang Y, Yan C, Gong X, et al. Molecular mechanism for inhibition of a critical component in the Arabidopsis thaliana abscisic acid signal transduction pathways, SnRK2.6, by protein phosphatase ABI1. J Biol Chem 2012; 287: 794-802.

123 Ng LM, Soon FF, Zhou XE, West GM, Kovach A, Suino-Powell KM, et al. Structural basis for basal activity and autoactivation of abscisic acid (ABA) signaling SnRK2 kinases. Proc Natl Acad Sci U S A 2011; 108: 21259-64.

124 Yunta C, Martinez-Ripoll M, Zhu JK, Albert A. The structure of Arabidopsis thaliana OST1 provides insights into the kinase regulation mechanism in response to osmotic stress. J Mol Biol 2011; 414: 135-44.

125 Bayliss R, Sardon T, Vernos I, Conti E. Structural basis of Aurora-A activation by TPX2 at the mitotic spindle. Mol Cell 2003; 12: 85162.

126 Jeffrey PD, Russo AA, Polyak K, Gibbs E, Hurwitz J, Massague J, et al. Mechanism of CDK activation revealed by the structure of a cyclinACDK2 complex. Nature 1995; 376: 313-20.
127 Kannan N, Haste N, Taylor SS, Neuwald AF. The hallmark of AGC kinase functional divergence is its C-terminal tail, a cis-acting regulatory module. Proc Natl Acad Sci U S A 2007; 104: 1272-7.

128 Lamers MB, Antson AA, Hubbard RE, Scott RK, Williams DH. Structure of the protein tyrosine kinase domain of C-terminal SrC kinase (CSK) in complex with staurosporine. J Mol Biol 1999; 285: 713-25.

129 Marx A, Nugoor C, Panneerselvam S, Mandelkow E. Structure and function of polarity-inducing kinase family MARK/Par-1 within the branch of AMPK/Snf1-related kinases. FASEB J 2010; 24: 1637-48.

130 Nolen B, Taylor S, Ghosh G. Regulation of protein kinases; controlling activity through activation segment conformation. Mol Cell 2004; 15: $661-75$.

131 Zhang X, Gureasko J, Shen K, Cole PA, Kuriyan J. An allosteric mechanism for activation of the kinase domain of epidermal growth factor receptor. Cell 2006; 125: 1137-49.

132 Vlad F, Droillard MJ, Valot B, Khafif M, Rodrigues A, Brault M, et al. Phospho-site mapping, genetic and in planta activation studies reveal key aspects of the different phosphorylation mechanisms involved in activation of SnRK2s. Plant J 2010; 63: 778-90.

133 Chen L, Jiao ZH, Zheng LS, Zhang YY, Xie ST, Wang ZX, et al. Structural insight into the autoinhibition mechanism of AMP-activated protein kinase. Nature 2009; 459: 1146-9.

134 Kornev AP, Haste NM, Taylor SS, Eyck LF. Surface comparison of active and inactive protein kinases identifies a conserved activation mechanism. Proc Natl Acad Sci U S A 2006; 103: 17783-8.

135 Pavletich NP. Mechanisms of cyclin-dependent kinase regulation: structures of Cdks, their cyclin activators, and Cip and INK4 inhibitors. J Mol Biol 1999; 287: 821-8.

136 Mosquna A, Peterson FC, Park SY, Lozano-Juste J, Volkman BF, Cutler SR. Potent and selective activation of abscisic acid receptors in vivo by mutational stabilization of their agonist-bound conformation. Proc Natl Acad Sci U S A 2011; 108: 20838-43.

137 Pizzio GA, Rodriguez L, Antoni R, Gonzalez-Guzman M, Yunta C, Merilo E, et al. The PYL4 A194T mutant uncovers a key role of PYR1LIKE4/PROTEIN PHOSPHATASE 2CA interaction for abscisic acid signaling and plant drought resistance. Plant Physiol 2013; 163 : 441-55.

138 Todoroki Y, Hirai N. Abscisic acid analogs for probing the mechanism of abscisic acid reception and inactivation. In: Atta-ur R ed. Studies in Natural Products Chemistry: Elsevier 2002: 321-60

139 Jia W, Zhang J, Zhang DP. Metabolism of xylem-delivered ABA in relation to $A B A$ flux and concentration in leaves of maize and Commelina communis. J Exp Bot 1996; 47: 1085-91.

140 Sharma N, Abrams SR, Waterer DR. Evaluation of abscisic acid analogs as holding agents for bedding plant seedlings. HortTechnology 2006; 16: 71-7.

141 Todoroki Y, Hirai N, Ohigashi H. Synthesis, biological activity and metabolism of (S)-(+)-3'-Fluoroabscisic acid. Tetrahedron 1995; 51: 6911-26.

142 Cutler AJ, Rose PA, Squires TM, Loewen MK, Shaw AC, Quail JW, et al. Inhibitors of Abscisic Acid 8'-Hydroxylase. Biochemistry 2000; 39: 13614-24.

143 Todoroki Y, Sawada M, Matsumoto M, Tsukada S, Ueno K, Isaka M, et al. Metabolism of $5^{\prime} \alpha, 8^{\prime}$-cycloabscisic acid, a highly potent and long-lasting abscisic acid analogue, in radish seedlings. Bioorg Med Chem 2004; 12: 363-70.

144 Cao M, Liu X, Zhang Y, Xue X, Zhou XE, Melcher K, et al. An ABAmimicking ligand that reduces water loss and promotes drought resistance in plants. Cell Res 2013; 23: 1043-54. 